\title{
Distribution and Sources of Polychlorinated Biphenyls in Air, Dust, and Sediment from India
}

\author{
Yogita Nayak ${ }^{1}$; Yaman Kumar Sahu²; Khageshwar Singh Patel ${ }^{3}$; Saroj Sharma ${ }^{4}$; Chin-Chang Hung ${ }^{5}$; \\ Pablo Martín-Ramos ${ }^{6}$; and Sema Yurdakul ${ }^{7}$
}

\begin{abstract}
Persistent organic pollutants, such as polychlorinated biphenyls (PCBs), pose a serious risk for human health and the environment. In this study, PCBs contamination and sources of ambient air, road dust, and sediments in the most polluted city in India, Raipur has been measured over the period 2008-2015. The seasonal variations of particulate matter (PM), elemental carbon (EC), organic carbon (OC), and carbonate carbon (CC) were studied, and maximum concentrations were detected in the December-January period each year. Total PCBs concentrations in the ambient air (associated road dust, and sediments samples during 2008 were in the $186-645 \mathrm{pg} \mathrm{m}^{-3}$, 102-537, and 241-538 $\mathrm{ng} \mathrm{g}^{-1}$ range, respectively. 2-chlorobiphenyl (PCB-1) and 4-chlorobiphenyl (PCB-3) were the dominant chemical compounds identified. A substantial vertical migration of the PCBs in the sediment was observed. Concentration variations (spatial and temporal), correlations, and sources of PCB are discussed. In particular, an average increment rate of $6.2 \%, 4.9 \%$, and $5.4 \%$ of PCBs concentration in the particulate matter $\left(\mathrm{PM}_{10}\right)$, road dust, and sediments respectively, was observed over the 2008-2015 period. The reported data points to India's low degree of accomplishment of the Stockholm Convention's requirement to phase out the use of PCBs in equipment by 2025 and ensure elimination of PCBs by 2028 .
\end{abstract}

Author keywords: PCBs; Contamination; Air; Dust \& sediment; Toxicity; Sources.

\section{Introduction}

Persistent organic pollutants (POPs)-fi.e., aldrin, chlordane, DDT, dieldrin, endrin, heptachlor, hexachlorobenzene, mirex, toxaphene, polychlorinated biphenyls (PCBs), polychlorinated dibenzo-p-dioxins (PCDD), and polychlorinated dibenzofurans (PCDF), plus the 16 so-called "new POPs" under the Stockholm Convention, are characterized by their high half-lives, low water solubility, and high lipid solubility (Harrad 2009). In particular, PCBs, a group of man-made oily liquids or solids, are widely used in electrical equipment, hydraulic fluids, heat transfer fluids, coolants, lubricants, and plasticizers in paint, paper, and plastics due to their low electrical conductivity and high

${ }^{1}$ School of Studies in Chemistry, Pt. Ravishankar Shukla Univ., Raipur, CG 492010, India. Email: nayakyogita@gmail.com

${ }^{2}$ School of Studies in Chemistry, Pt. Ravishankar Shukla Univ., Raipur, CG 492010, India. Email: yamankumarsahu8@gmail.com

${ }^{3}$ Research \& Development, Amity Univ., State Highway-9, Manth (Kharora), Raipur, CG 493225, India (corresponding author). Email: patelkhageshwarsingh@mail.com

${ }^{4}$ Dept. of Chemistry, Government Nagarjuna Post Graduate College of Science, Raipur, CG 492002, India. Email: ssharmagr8@gmail.com

${ }^{5}$ Institute of Marine Environmental Chemistry and Ecology, National Taiwan Ocean Univ., Keelung 20224, Taiwan. Email: cchung@mail.ntou .edu.tw

${ }^{6}$ Instituto de Investigación en Ciencias Ambientales (IUCA), EPS, Univ. of Zaragoza, Carretera de Cuarte s/n, 22071 Huesca, Spain. resistance to heat and thermal degradation (Robertson and Hansen 2001). PCBs are a class of aromatic compounds two benzene rings with a maximum of 10 substituted $\mathrm{Cl}$-atoms, and are mixtures of up to 209 individual chlorinated compounds, known as congeners, whose chemistry differs from species to species (Hutzinger 1974). PCBs have been reported to cause many adverse effects both on wildlife and on human health, including immune deficiency, nervous system alteration, endocrine disruption, and gastrointestinal system bleeding and liver damage (ATSDAR 2014).

Contamination with PBCs has been reported in air, water, soil, dust, and sediment samples from all over the world (Anh et al. 2019; Biterna and Voutsa 2005; Chakraborty et al. 2013, 2016; Devi et al. 2014; Goel et al. 2016; Kim and Masunaga 2005; Kumar et al. 2011; Nasir et al. 2014; Sakin and Tasdemir 2016; Syed et al. 2013; Wang et al. 2013). In fact, at present, the United Nations Environment Program (UNEP) estimates that $83 \%$ of the total amount of PCBs in the world ( $c a .14$ million tons) still remains to be eliminated.

In the particular case of India, the Stockholm Convention was ratified in 2002 and entered into force in 2006. Preliminary investigations on PCBs contamination in sediments and sludge from the Raipur area been previously reported (Patel et al. 2013, 2015): Findings of the highest concentrations of the monochlorobipheny1 (MCBP) congeners were reported to date. Nonetheless, as noted by the PCB Elimination Network (PEN), "data on elimination of PCB is often incomprehensive, outdated, and incomplete (United Nations Environmental Programme, UNITAR 2017)." Consequently, the aim of present work is to describe the distribution, variation, sources, and fate of PCBs congeners in different environmental samples (air, dust, and sediment) from the most industrialized area of central India, Raipur city (capital of Chhattisgarh state), to strengthen the analyses of India's PCBs situation, in line with the "country specific diagnoses" PEN key theme. 


\section{Materials and Methods}

\section{Study Area}

The capital eity of Chhattisgarh state, Raipur $\left(21^{\circ} 23^{\prime} \mathrm{N}, 81^{\circ} 63^{\prime} \mathrm{E}\right)$, with a population of $c a$. 2 million inhabitants, was selected for the proposed investigation due to its high degree of industrialization. Raipur city and the nearby region are home to many coal, power, steel, and aluminum industries. For instance, Asia's biggest steel plant is located in Bhilai, and Korba (known as the "power capital of India") is heavily polluted by thermal power plants (Jaiswal et al. 2019a, b).

\section{Environmental Samples Collection}

The sampling of the particulate matter was carried out for 13 months (February 2007 to January 2008) in Kota, a residential area (Fig. 1). For spatial variation studies, the sampling was carried out at three additional locations: Pt. Ravishankar Shukla University, Amapara, and Raipura in December 2008. These sampling sites were approximately $2 \mathrm{~km}$ distant from each other. The locations at Kota and Amapara commercial and traffic sites. The distance of these sampling sites from the industrial area was approximately $2 \mathrm{~km}$ to the east. For temporal variation studies, Kota site was selected, and one $\mathrm{PM}_{10}$ sample was collected every January from 2009 to 2015.

A Partisol model 2300 sequential speciation air sampler (Thermo Scientific, Waltham, MA, USA) was used for the collection of particulate matter (PM) samples. Coarse particles with a diameter $<10 \mu \mathrm{m}\left(\mathrm{PM}_{10}\right)$ were collected on dried Whatman QM-A 47-mm quartz fiber filters, housed in molded filter cassettes. The sampler was installed on the roofs of buildings at approximately $10 \mathrm{~m}$ above the ground level. The sampling was carried out for $24 \mathrm{~h}$ (6 a.m.-6 a.m.). Similarly, a relevant field sample blank was prepared, as the filter paper was exposed to the environment during the mounting and dismounting period. The mass difference $\left(w_{2}-w_{1}\right)$ of the dried blank filter paper before $\left(w_{1}\right)$ and after the exposure period $\left(w_{2}\right)$ was evaluated and subtracted from the sample mass.

The road dust samples were collected using a stainless-steel scoop from eight locations along Raipur city's highway: Tatibandh, Hirapur, Sarora, Khamtarai, Birgaon, Urla, Sankra, and Siltara, during May 2008. These examined sites were situated in an area of approximate $10 \mathrm{~km}$ radius. The last four sites listed were located in the industrial area. The Khamtarai site was located in a heavy traffic area. The sample was collected from both sides (left and right) of the road junction in two $250-\mathrm{mL}$ glass bottles. These samples were mixed in equal mass ratio to form a composite sample. Similarly, the sampling was extended up to period 2015, and one sample was taken every year in May from 2009 to 2015 at the Khamtarai site for the temporal variation studies. The dust samples were sundried for one week, and further dried at $50^{\circ} \mathrm{C}$ in a hot air oven overnight. The samples were then crushed into fine-powder form by sieving out of particles of mesh size $>100 \mu \mathrm{m}$ and finally stored in an aluminum foil for the analyses.

Ten surface sediment samples $(0-10 \mathrm{~cm}$ depth) were collected in May 2008 from 10 ponds (Rohnipuram, Ashi, Budheshwar, Raja, Pandri, Siltara, Urkura, Birgoan, and Sarora) of Raipur city. These ponds were situated over an area of approximately $25 \mathrm{~km}^{2}$. The last five listed ponds were located at industrial sites. For depth studies, two more sediment samples at a depth of 10-20 and 20$30 \mathrm{~cm}$ were collected in Siltara in 2008. The Raja pond was chosen for the temporal evolution investigation, and one sample was collected every year in May from 2009 to 2015.
A polyvinyl chloride (PVC) stormwater pipe of 5-cm radius (Simpson and Batley 2016) was used for the sediment collection. The samples were collected from five sites of the pond (east, west, north, and south corners, and midpoint). These samples were mixed equally to prepare a composite sample. They were dried, crushed, sieved, and stored in a similar manner to the road dust samples in a dust-free laboratory.

\section{Carbon Speciation}

Elemental carbon (EC), organic carbon (OC), and carbonate carbon (CC) were analyzed in the particulate matter, road dust, and sediment samples. An elemental ECS 4010 CHNSO analyzer (Costech Analytical Technologies Inc, USA) was used for the analysis of total carbon (TC). The dust samples were oxidized with $\mathrm{O}_{2}$ at $1,020^{\circ} \mathrm{C}$ using a-constant helium-flow as a carrier. The resulting $\mathrm{CO}_{2}$ gas was detected by a thermal conductivity detector. The $\mathrm{CC}$ content was removed by treating the sample with $\mathrm{HCl}$ acid in a $\mathrm{CO}_{2}$-free atmosphere. The $\mathrm{EC}$ and $\mathrm{OC}$ contents were determined by the thermal method deseribed. The OC content was analyzed by titration method using $\mathrm{K}_{2} \mathrm{Cr}_{2} \mathrm{O}_{7}$ as oxidant. The $\mathrm{EC}$ content in the sample was evaluated by subtracting the $\mathrm{CC}$ and $\mathrm{OC}$ values from the TC content using

$$
\mathrm{EC}=\mathrm{TC}-(\mathrm{CC}+\mathrm{OC})
$$

where EC, CC, OC, and TC stand for the content of elemental carbon, carbonate carbon, organic carbon, and total carbon, respectively.

\section{PCBs Analysis}

The analyte sample was dried with sodium sulfate and extracted using a Dionex accelerated solvent extraction (ASE) system. The surrogate standards $\left({ }^{13} \mathrm{C}_{12}\right.$-labelled $\left.\mathrm{PCBs}\right)$ were added and the samples were extracted with methylene chloride as a solvent. The extract was treated with copper to remove sulfur, and was purified by silica/alumina column chromatography to isolate the PCB fractions (Wade et al. 1988). The quantitative analyses were performed with a HP 5890 gas chromatograph (Agilent Technologies, Santa Clara, CA, USA), and a HP 5970 mass spectrometer in the SIM mode for PCBs, according to the method described by Sericano (2002). The quantification of the PCBs was based on the primary ion with supplementary monitoring of two additional masses for each analyte to verify the peaks identification.

\section{Quality Assurance/Quality Control Analysis}

One-way analysis of variance (ANOVA) was performed to analyze the distribution variability of the PCBs concentrations in the particulates, road dust, and sediments. IBM-SPSS 20.0 (SPSS Inc..) software was employed for the factor analysis of source contributions of PCBs in the air, dust, and sediment by extracting factors with an Eigenvalue of $>1.0$ (Shyu et al. 2011).

The surrogate standard $\left({ }^{13} \mathrm{C}_{12}\right.$-labelled PCBs) was used to determine the extraction efficiency for the targeted PCB congeners. The standard was added prior to the extraction processes, and the average recoveries of these standards from the different environmental matrices varied from $91.1 \%$ to $102 \%$. The calibration curve was prepared by injections of standard solutions containing a mixture of the PCBs at four concentration levels. The blanks used were clear of all the examined PCBs. The limits of detection (LODs) and quantification (LOQs) were determined. Reported values are expressed as an average across three replicate measurements, both for the carbon and PCB analyses. 

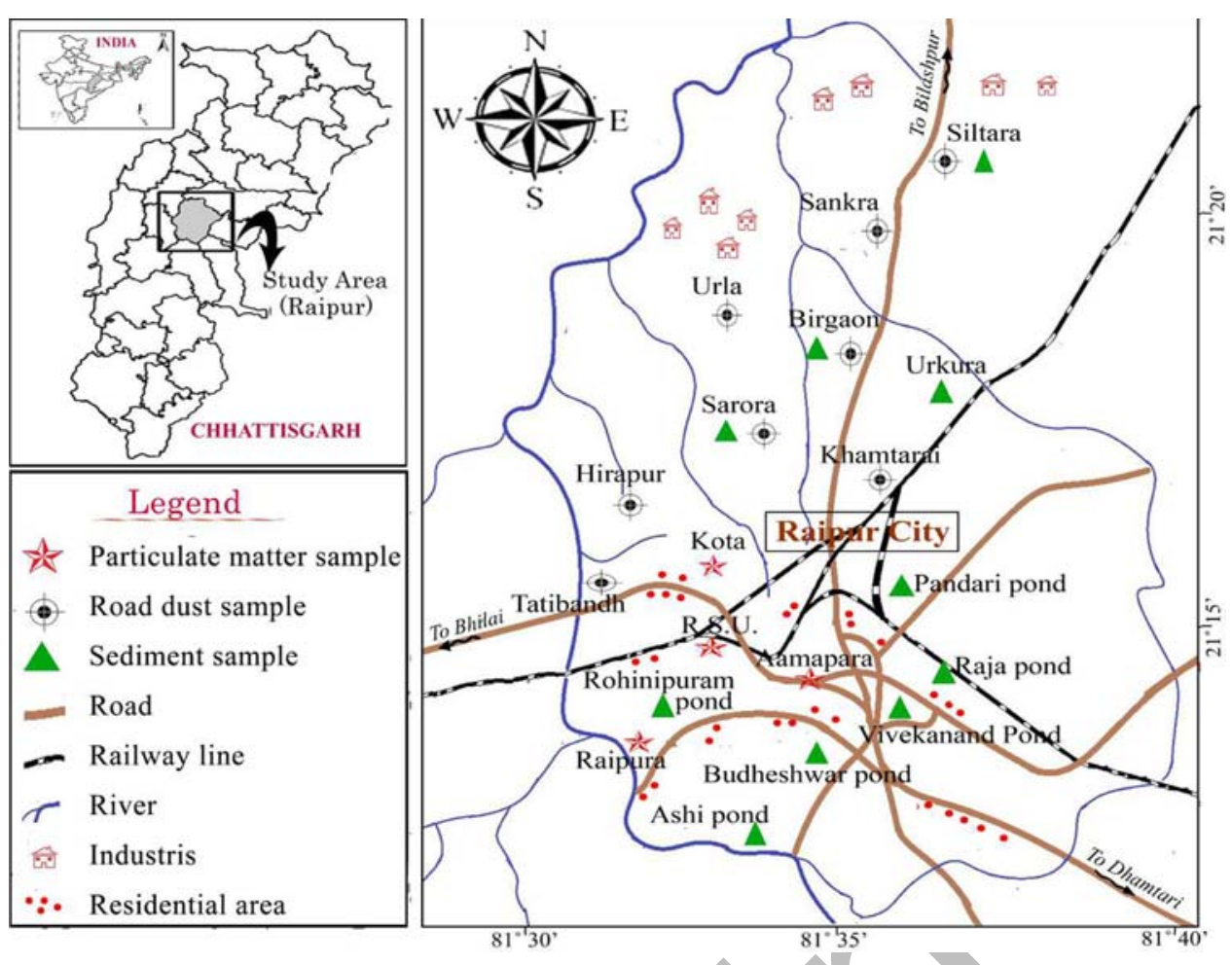

Fig. 1. Sample locations for collection of particulates, road dusts, and sediments in Raipur city. (Map by Yaman Kumar Sahu-)

\section{Results and Discussion}

\section{Meteorology and Concentrations of Particulates, Carbon, and PCBs in Air}

The meteorology (i.e., temperature, humidity, vapor pressure, wind speed, and sunshine) for the study period February 2007 to January 2008 ranged from $19.7^{\circ} \mathrm{C}$ to $38.1^{\circ} \mathrm{C}, 20.8 \%$ to $92.8 \%, 7.1$ to $24.5 \mathrm{~mm}, 1.5$ to $12 \mathrm{~km} \mathrm{~h}^{-1}$, and 0.9 to $10 \mathrm{~h} \mathrm{day}^{-1}$, respectively. The lowest values of the ambient temperature, vapor pressure, wind speed, and sunshine were observed from December to January. A total rainfall of $96 \mathrm{~cm}$, together with the maximum humidity, was registered from July to September 2007. The winds blew from the northeast and showed speeds of approximately $11 \mathrm{~km}$ during the June to August 2007 period. The lowest wind speed from the N direction was observed during the October 2007 to January 2008 period.

The major fraction of the PM was composed of carbons: EC, $\mathrm{OC}$, and CC. The concentration of $(n=24) \mathrm{PM}_{10}, \mathrm{EC}_{10}, \mathrm{OC}_{10}$, and $\mathrm{CC}_{10}$ in the air during the February 2007 to January 2008 period ranged from 116 to 523 , from 8.8 to 65.5 , from 7.2 to 55.4 , and from 6.1 to $58.7 \mu \mathrm{g} \mathrm{m}^{-3}$, with mean values of $283 \pm 138,28.8 \pm$ $16.9,23.2 \pm 13.6$, and $22.7 \pm 16.9 \mu \mathrm{g} \mathrm{m}^{-3}$, respectively. The PM showed a negative correlation with the meteorological factors (i.e., rain-fall, temperature, humidity, vapor pressure, and wind speed) of $r=-0.31-0.74$. Three meteorological factors (rain, wind speed, and wind direction) markedly influenced the PM concentration. The highest mass concentration was observed in the winter season, December-January, expected due to the lowest atmospheric pressure, temperature, and wind speed. In turn in the rainy season (June-August), the lowest concentration was recorded, probably due to PM washout with the rain.

A similar distribution trend of the PM and carbons in the Raipur city was reported by Jaiswal et al. (2019b). In the hilly area of north India, the different distribution trend of the PM and carbons (maximal in the summer season) likely due to precipitation of the particulates with the snow in the winter season (Ganguly et al. 2019).

Polychlorinated biphenyls are considered as significant environmental air contaminants due to their ecotoxicological and human health implications. The PM in the air is associated with large fraction of EC, OC, and CC. The concentration of the $\mathrm{PM}_{10}$, $\mathrm{EC}, \mathrm{OC}$, and $\mathrm{CC}$ in the ambient air of Raipur city monitored at four locations (Kota, Pt. R. S. University, Raipura Chowk, and Amapara) were in the 388-845, 33-81, 23-52, and 28-65 $\mu \mathrm{g} \mathrm{m}^{-3}$ range, respectively, with average values of $574 \pm 222,53 \pm 23,36 \pm 14$, and $44 \pm 18 \mu \mathrm{g} \mathrm{m}^{-3}$, respectively. The highest $\mathrm{PM}_{10}$ and carbon concentrations in the air corresponded to samples collected in Amapara, most probably due to vehicular emissions.

The concentrations of the PCBs congeners in the ambient air associated the particulates $\left(\mathrm{PM}_{10}\right)$ are presented in Table 1. A total 83 congeners in the ambient air-were detected. The total concentration of PCBs $(n=4)$ ranged from 72.07 to $429.57 \mathrm{pg} \mathrm{m}^{-3}$, with a mean value of $247.54 \mathrm{pg} \mathrm{m}^{-3}$. The maximum PCBs value was found for the Kota site, expected due to vehicular emissions. The dominant congeners $(1,3$, and 17$)$ were found at concentrations in the 2.4-130.41, 49.31-147.74, and $1.42-18.8 \mathrm{pg} \mathrm{m}^{-3}$ ranges, with mean values of $46.97,96.10$, and $9.01 \mathrm{pg} \mathrm{m}^{-3}$, respectively.

Table 1 summarizes the concentration of individual congeners in the air. Total concentrations of MCBs, dichlorobiphenyls (DCBs), trichlorobiphenyls (TCBs), tetrachlorobiphenyls (TeCBs), pentachlorobiphenyls (PeCBs), hexachlorobiphenyls (HCBs), heptachlorobiphenyls (HeCBs), octachlorobiphenyls (OCBs), and nonachlorobiphenyls (NCBs) examined in the ambient air varied from 53.16 to 278.15 , from 4.44 to 37.50 , from 2.57 to 33.49 , from 1.81 to 57.46 , from 6.65 to 30.85 , from 3.02 to 27.24 , from 2.19 to 11.87 , from 0.16 to 2.45 , and from 0.64 to $2.14 \mathrm{pg} \mathrm{m}^{-3}$, respectively, with the highest and the lowest values for MCBs (143.06 \pm $\left.99.47 \mathrm{pg} \mathrm{m}^{-3}\right)$ and NCBs $\left(1.12 \pm 0.70 \mathrm{pg} \mathrm{m}^{-3}\right)$, respectively. The MCBs accounted for $54.2 \%$ of the total PCBs concentration in the air. The other congeners (DCBs, TCBs, TeCBs, PeCBs, HCBs, 
Table 1. PCB concentration in ambient air and particulates collected in 2008

\begin{tabular}{|c|c|c|c|c|c|c|c|c|c|c|c|c|}
\hline \multirow[b]{2}{*}{ S. no. } & \multirow[b]{2}{*}{ Type } & \multirow[b]{2}{*}{ Congener } & \multicolumn{5}{|c|}{ Ambient air, $\mathrm{pg} \mathrm{m}^{-3}$} & \multicolumn{5}{|c|}{ Ambient particulate $\left(\mathrm{PM}_{10}\right), \mathrm{ng} \mathrm{g}^{-1}$} \\
\hline & & & Kota & RSU & Raipura & Amapara & Mean value \pm STD & Kota & RSU & Raipura & Amapara & Mean value \pm STD \\
\hline 1 & MCBs & 1 & 130.41 & 2.4 & 37.01 & 18.05 & $46.97 \pm 57.40$ & 196.40 & 6.19 & 337.53 & 21.36 & $140.37 \pm 157.25$ \\
\hline 2 & & 3 & 147.74 & 50.76 & 49.31 & 136.57 & $96.10 \pm 53.38$ & 222.50 & 130.82 & 382.87 & 161.62 & $224.45 \pm 112.27$ \\
\hline 3 & DCBs & $4+10$ & 0.49 & 0.05 & 0.69 & 1.96 & $0.80 \pm 0.82$ & 0.74 & 0.13 & 1.26 & 2.32 & $1.11 \pm 0.93$ \\
\hline 4 & & 6 & 0 & 0 & 0 & 0.06 & $0.02 \pm 0.03$ & 0.00 & 0.00 & 0.00 & 0.07 & $0.02 \pm 0.04$ \\
\hline 5 & & $7+9$ & 0.01 & 0.03 & 0.14 & 0 & $0.05 \pm 0.06$ & 0.02 & 0.08 & 0.00 & 0.00 & $0.03 \pm 0.04$ \\
\hline 6 & & $8+5$ & 3.51 & 1.79 & 2.87 & 3.45 & $2.91 \pm 0.80$ & 5.29 & 4.61 & 9.07 & 4.08 & $5.76 \pm 2.26$ \\
\hline 7 & TCBs & $16+32$ & 0 & 0 & 0 & 0.45 & $0.11 \pm 0.23$ & 0.00 & 0.00 & 0.00 & 0.53 & $0.13 \pm 0.27$ \\
\hline 8 & & 17 & 18.8 & 1.42 & 9.43 & 6.39 & $9.01 \pm 7.31$ & 28.31 & 3.66 & 48.87 & 7.56 & $22.10 \pm 20.87$ \\
\hline 9 & & 18 & 10.45 & 0 & 2.86 & 0 & $3.33 \pm 4.94$ & 15.74 & 0.00 & 27.20 & 0.00 & $10.74 \pm 13.25$ \\
\hline 10 & & 19 & 0.06 & 0.1 & 0.06 & 0.05 & $0.07 \pm 0.02$ & 0.09 & 0.26 & 0.25 & 0.06 & $0.17 \pm 0.10$ \\
\hline 11 & & $21+33+53$ & 0.2 & 0.04 & 1.00 & 0.57 & $0.45 \pm 0.43$ & 0.30 & 0.10 & 0.50 & 0.67 & $0.39 \pm 0.25$ \\
\hline 12 & & 22 & 1.12 & 0.16 & 1.11 & 2.15 & $1.14 \pm 0.81$ & 1.69 & 0.41 & 3.02 & 2.54 & $1.92 \pm 1.14$ \\
\hline 13 & & 24 & 2.13 & 0.33 & 0.83 & 1.52 & $1.20 \pm 0.79$ & 3.21 & 0.85 & 5.54 & 1.80 & $2.85 \pm 2.04$ \\
\hline 14 & & 25 & 0.21 & 0 & 0.09 & 0.28 & $0.15 \pm 0.12$ & 0.32 & 0.00 & 0.50 & 0.33 & $0.29 \pm 0.21$ \\
\hline 15 & & $28+31$ & 0.18 & 0.28 & 1.23 & 1.13 & $0.71 \pm 0.55$ & 0.27 & 0.72 & 0.50 & 1.34 & $0.71 \pm 0.46$ \\
\hline 16 & & 29 & 0.34 & 0.24 & 0.26 & 0.19 & $0.26 \pm 0.06$ & 0.51 & 0.62 & 1.01 & 0.22 & $0.59 \pm 0.33$ \\
\hline 17 & TeCBs & $37+42$ & 3.61 & 0 & 1.34 & 4.62 & $2.39 \pm 2.10$ & 5.44 & 0.00 & 9.32 & 5.47 & $5.06 \pm 3.83$ \\
\hline 18 & & 40 & 2.03 & 0.07 & 0.69 & 2.34 & $1.28 \pm 1.08$ & 3.06 & 0.18 & 5.29 & 2.77 & $2.83 \pm 2.09$ \\
\hline 19 & & $41+64+71$ & 2.26 & 0.07 & 0.07 & 2.32 & $1.18 \pm 1.28$ & 3.40 & 0.18 & 5.79 & 2.75 & $3.03 \pm 2.31$ \\
\hline 20 & & 44 & 0.78 & 0 & 0.27 & 1.64 & $0.67 \pm 0.72$ & 1.17 & 0.00 & 2.02 & 1.94 & $1.28 \pm 0.94$ \\
\hline 21 & & 45 & 0.41 & 0.06 & 0 & 0.47 & $0.24 \pm 0.24$ & 0.62 & 0.15 & 1.01 & 0.56 & $0.59 \pm 0.35$ \\
\hline 22 & & 46 & 0.71 & 0 & 0.5 & 1.37 & $0.65 \pm 1.49$ & 1.07 & 0.00 & 1.76 & 1.62 & $1.11 \pm 0.80$ \\
\hline 23 & & $47+48$ & 1.34 & 0.2 & 0.97 & 3.66 & $1.54 \pm 1.49$ & 2.02 & 0.52 & 3.53 & 4.33 & $2.60 \pm 1.69$ \\
\hline 24 & & 49 & 0.72 & 0.48 & 0 & 2.05 & $0.81 \pm 0.88$ & 1.08 & 1.24 & 1.76 & 2.43 & $1.63 \pm 0.61$ \\
\hline 25 & & 51 & 0 & 0 & 0 & 0.48 & $0.12 \pm .24$ & 0.00 & 0.00 & 0.00 & 0.57 & $0.14 \pm 0.29$ \\
\hline 26 & & $56+60$ & 14.32 & 0.63 & 2.99 & 13.07 & $7.75 \pm 6.95$ & 21.57 & 1.62 & 37.03 & 15.47 & $18.92 \pm 14.68$ \\
\hline 27 & & 62 & 0.71 & 0.14 & 0.20 & 1.42 & $0.62 \pm 0.59$ & 1.07 & 0.36 & 1.76 & 1.68 & $1.22 \pm 0.65$ \\
\hline 28 & & 63 & 1.8 & 0.11 & 1.20 & 2.47 & $1.40 \pm 1.00$ & 2.71 & 0.28 & 4.79 & 2.92 & $2.68 \pm 1.85$ \\
\hline 29 & & $66+95$ & 5.31 & 0 & 2.43 & 5.24 & $3.25 \pm 2.55$ & 8.00 & 0.00 & 13.85 & 6.20 & $7.01 \pm 5.70$ \\
\hline 30 & & 70.76 & 11.28 & 0.03 & 5.66 & 11.47 & $7.11 \pm 5.44$ & 16.99 & 0.08 & 29.22 & 13.57 & $14.97 \pm 11.98$ \\
\hline 31 & & 74 & 4.72 & 0.02 & 2.55 & 484 & $3.03 \pm 2.27$ & 7.11 & 0.05 & 12.34 & 5.73 & $6.31 \pm 5.05$ \\
\hline 32 & $\mathrm{PeCBs}$ & $77+110$ & 1.06 & 0.9 & 3.17 & 2.96 & $2.02 \pm 1.21$ & 1.60 & 2.32 & 2.77 & 3.50 & $2.55 \pm 0.80$ \\
\hline 33 & & $81+87$ & 0.55 & 0 & 0.48 & 1.96 & $0.75 \pm 0.84$ & 0.83 & 0.00 & 1.51 & 2.32 & $1.17 \pm 0.99$ \\
\hline 34 & & $82+151$ & 1.96 & 1.69 & 10.5 & 1.03 & $3.80 \pm 4.49$ & 2.95 & 4.36 & 5.04 & 1.22 & $3.39 \pm 1.69$ \\
\hline 35 & & 83 & 1.25 & 0 & 0.77 & 1.59 & $0.90 \pm 0.69$ & 1.88 & 0.00 & 3.27 & 1.88 & $1.76 \pm 1.34$ \\
\hline 36 & & 85 & 1.43 & 1.03 & 4.23 & 0.78 & $1.87 \pm 1.60$ & 2.15 & 2.65 & 3.78 & 0.92 & $2.38 \pm 1.19$ \\
\hline 37 & & 89 & 6.28 & 0 & 0.97 & 4.74 & $3.00 \pm 2.99$ & 9.46 & 0.00 & 16.37 & 5.61 & $7.86 \pm 6.88$ \\
\hline 38 & & 91 & 0.75 & 0.81 & 1.01 & 3.45 & $1.51 \pm 1.30$ & 1.13 & 2.09 & 2.02 & 4.08 & $2.33 \pm 1.25$ \\
\hline 39 & & $92+84$ & 2.23 & 0.02 & 0.07 & 1.88 & $1.05 \pm 1.17$ & 3.36 & 0.05 & 5.79 & 2.22 & $2.86 \pm 2.39$ \\
\hline 40 & & 97 & 1.19 & 0 & 1.59 & 1.86 & $1.16 \pm 0.82$ & 1.79 & 0.00 & 3.02 & 2.20 & $1.75 \pm 1.28$ \\
\hline 41 & & 99 & 2.83 & 0 & 0.26 & 2.73 & $1.46 \pm 1.53$ & 4.26 & 0.00 & 7.30 & 3.23 & $3.70 \pm 3.01$ \\
\hline 42 & & 100 & 1.7 & 0.96 & 0.54 & 1.71 & $1.23 \pm 0.58$ & 2.56 & 2.47 & 4.53 & 2.02 & $2.90 \pm 1.12$ \\
\hline 43 & & 101 & 5.22 & 0 & 1.57 & 4.86 & $2.91 \pm 2.54$ & 7.86 & 0.00 & 13.60 & 5.75 & $6.80 \pm 5.62$ \\
\hline 44 & & 105 & 1.2 & 0.09 & 1.61 & 1.03 & $0.98 \pm 0.64$ & 1.81 & 0.23 & 3.02 & 1.22 & $1.57 \pm 1.17$ \\
\hline 45 & & 107 & 0.54 & 0.99 & 0.65 & 0.02 & $0.55 \pm 0.40$ & 0.81 & 2.55 & 1.51 & 0.02 & $1.22 \pm 1.07$ \\
\hline 46 & & 118 & 0.32 & 0.16 & 0.32 & 0.22 & $0.26 \pm 0.08$ & 0.48 & 0.41 & 0.76 & 0.26 & $0.48 \pm 0.21$ \\
\hline 47 & & $123+149$ & 1.63 & 0 & 0.07 & 0.03 & $0.43 \pm 0.80$ & 2.45 & 0.00 & 4.28 & 0.04 & $1.69 \pm 2.07$ \\
\hline 48 & $\mathrm{HCBs}$ & 132 & 0.86 & 0 & 1.22 & 0.85 & $0.73 \pm 0.52$ & 1.30 & 0.00 & 2.27 & 1.01 & $1.15 \pm 0.93$ \\
\hline 49 & & 134 & 0.82 & 0 & 0.88 & 0.57 & $0.57 \pm 0.40$ & 1.23 & 0.00 & 2.02 & 0.67 & $0.98 \pm 0.86$ \\
\hline 50 & & $135+144$ & 0 & 0 & 1.5 & 3.04 & $1.14 \pm 1.45$ & 0.00 & 0.00 & 0.00 & 3.60 & $0.90 \pm 1.80$ \\
\hline 51 & & 136 & 0.82 & 1.71 & 5.1 & 4.01 & $2.91 \pm .98$ & 1.23 & 4.41 & 2.02 & 4.75 & $3.10 \pm 1.74$ \\
\hline 52 & & $137+130+176$ & 2.29 & 0 & 2.26 & 1.44 & $1.50 \pm 1.07$ & 3.45 & 0.00 & 6.05 & 1.70 & $2.80 \pm 2.58$ \\
\hline 53 & & $138+158$ & 7.37 & 0.37 & 7.36 & 8.03 & $5.78 \pm 3.62$ & 11.10 & 0.95 & 19.14 & 9.50 & $10.17 \pm 7.46$ \\
\hline 54 & & 141 & 0.31 & 0.05 & 1.59 & 1.34 & $0.82 \pm 0.76$ & 0.47 & 0.13 & 0.76 & 1.59 & $0.74 \pm 0.62$ \\
\hline 55 & & 146 & 0.33 & 0 & 1.27 & 0.07 & $0.42 \pm 0.59$ & 0.50 & 0.00 & 0.76 & 0.08 & $0.34 \pm 0.36$ \\
\hline 56 & & 153 & 3.62 & 0.89 & 4.97 & 3.18 & $3.17 \pm 1.70$ & 5.45 & 2.29 & 9.32 & 3.76 & $5.21 \pm 3.03$ \\
\hline 57 & & 157 & 0.9 & 0 & 0 & 3.07 & $0.99 \pm 1.45$ & 1.36 & 0.00 & 2.27 & 3.63 & $1.82 \pm 1.53$ \\
\hline 58 & & 163 & 0.47 & 0 & 1.09 & 0 & $0.39 \pm 0.52$ & 0.71 & 0.00 & 1.26 & 0.00 & $0.49 \pm 0.61$ \\
\hline 59 & $\mathrm{HeCBs}$ & $172+197$ & 0.92 & 0.31 & 0.19 & 0.72 & $0.54 \pm 0.34$ & 1.39 & 0.80 & 2.27 & 0.85 & $1.33 \pm 0.68$ \\
\hline 60 & & 174 & 0.7 & 0.11 & 0.14 & 0.67 & $0.41 \pm 0.32$ & 1.05 & 0.28 & 1.76 & 0.79 & $0.97 \pm 0.62$ \\
\hline 61 & & 177 & 1.4 & 0.07 & 0.28 & 1.36 & $0.78 \pm 0.70$ & 2.11 & 0.18 & 3.53 & 1.61 & $1.86 \pm 1.38$ \\
\hline
\end{tabular}


Table 1. (Continued.)

\begin{tabular}{|c|c|c|c|c|c|c|c|c|c|c|c|c|}
\hline \multirow[b]{2}{*}{ S. no. } & \multirow[b]{2}{*}{ Type } & \multirow[b]{2}{*}{ Congener } & \multicolumn{5}{|c|}{ Ambient air, $\mathrm{pg} \mathrm{m}^{-3}$} & \multicolumn{5}{|c|}{ Ambient particulate $\left(\mathrm{PM}_{10}\right), \mathrm{ng} \mathrm{g}^{-1}$} \\
\hline & & & Kota & RSU & Raipura & Amapara & Mean value \pm STD & Kota & RSU & Raipura & Amapara & Mean value \pm STD \\
\hline 62 & & $178+129$ & 1.03 & 0.57 & 1.16 & 0.86 & $0.91 \pm 0.25$ & 1.55 & 1.47 & 2.77 & 1.02 & $1.70 \pm 0.75$ \\
\hline 63 & & $180+193$ & 0.88 & 1.01 & 3.58 & 1.57 & $1.76 \pm 1.25$ & 1.33 & 2.60 & 2.27 & 1.86 & $2.02 \pm 0.55$ \\
\hline 64 & & 183 & 2.86 & 0 & 0.52 & 1.07 & $1.11 \pm 1.24$ & 4.31 & 0.00 & 7.30 & 1.27 & $3.22 \pm 3.27$ \\
\hline 65 & & 185 & 0.82 & 0 & 0.13 & 1.03 & $0.50 \pm 0.51$ & 1.23 & 0.00 & 2.02 & 1.22 & $1.12 \pm 0.83$ \\
\hline 66 & & $202+171+156$ & 3.26 & 0.12 & 0.73 & 1.71 & $1.46 \pm 1.37$ & 4.91 & 0.31 & 8.56 & 2.02 & $3.95 \pm 3.61$ \\
\hline 67 & OCBs & 194 & 0.12 & 0 & 0.04 & 0.03 & $0.05 \pm 0.05$ & 0.18 & 0.00 & 0.25 & 0.04 & $0.12 \pm 0.12$ \\
\hline 68 & & $196+203$ & 0.55 & 0 & 0.77 & 0.63 & $0.49 \pm 0.34$ & 0.83 & 0.00 & 1.51 & 0.75 & $0.77 \pm 0.62$ \\
\hline 69 & & 198 & 0.12 & 0 & 0.3 & 0.25 & $0.17 \pm 0.14$ & 0.18 & 0.00 & 0.25 & 0.30 & $0.18 \pm 0.13$ \\
\hline 70 & & 199 & 0.25 & 0 & 0 & 0 & $0.06 \pm 0.13$ & 0.38 & 0.00 & 0.76 & 0.00 & $0.29 \pm 0.36$ \\
\hline 71 & & 201 & 1.41 & 0.16 & 0.5 & 0.55 & $0.66 \pm 0.53$ & 2.12 & 0.41 & 3.78 & 0.65 & $1.74 \pm 1.56$ \\
\hline 72 & NCBs & 206 & 0.67 & 0.64 & 1.03 & 2.14 & $1.12 \pm 0.70$ & 1.01 & 1.65 & 1.76 & 2.53 & $1.74 \pm 0.62$ \\
\hline
\end{tabular}

Note: RSU = Ravishankar University; $\mathrm{MCBs}=$ monochlorobiphenyls; $\mathrm{DCBs}=$ dichlorobiphenyls; $\mathrm{TCBs}=$ trichlorobiphenyls; TeCBs = tetrachlorobiphenyls; $\mathrm{PCBs}=$ pentrachlorobiphenyls; $\mathrm{HCBs}=$ hexachlorobiphenyls; $\mathrm{HeCBs}=$ heptachlorobiphenyls; OCBs = octachlorobiphenyls; and NCBs = nonachlorobiphenyls.

$\mathrm{HeCBs}$, OCBs, and $\mathrm{NCBs}$ ) contributed the remaining $45.8 \%$ of the PCBs content.

A lower PCBs content was observed in Raipur city $(248 \pm$ $152 \mathrm{pg} \mathrm{m}^{-3}$ ) than the one reported for the ambient air in Kanpur city (254-432 $\mathrm{pg} \mathrm{m}^{-3}$ ) (Goel et al. 2016), although it was considerably higher than the contents reported (4-389 $\mathrm{pg} \mathrm{m}^{-3}$ ) in other locations, such as Japan, France, Atlantic Ocean, Korea, or Pakistan (Nasir et al. 2014; Syed et al. 2013; Baek et al. 2010; Gioia et al. 2008; Blanchard et al. 2006; Kim and Masunaga 2005).

\section{Concentration of Carbon and PCBs in Particulate, Dust, and Sediments}

EC, OC, and PCBs are emitted during various combustion and industrial processes (Brunciak et al. 2001) and are distributed in various environmental compartments (viz. air, water, dust, and soil) in urban and industrial areas (Malina and Mazlova 2017). Along roads, they are predominantly emitted by vehicular emissions (Liu et al. 2019). The environmental PCBs are transported to water reservoirs by rain, runoff water, industrial and municipal waste, etc. (Froese et al. 1997). Tables 1-3 summarize the environmental contamination of PCBs in the ambient particulates, road dust, and sediments of the study area.

The concentration of EC, OC, and CC in the coarse particulate matter $\left(\mathrm{PM}_{10}\right)$ ranged from $8.4 \%$ to $9.61 \%$, from $5.45 \%$ to $8.81 \%$, and from $7.54 \%$ to $9.43 \%$, respectively, with mean values of $9.15 \%$ $\pm 0.55 \%, 6.87 \% \pm 1.20 \%$, and $8.74 \% \pm 0.61 \%$, respectively. The comparable EC concentration in the road dust and sediments varied from $5.8 \%$ to $6.61 \%$ and from $7.29 \%$ to $7.77 \%$, with average values of $6.26 \% \pm 0.31 \%$ and $7.56 \% \pm 0.21 \%$. However, very low OC $(0.39 \% \pm 0.09 \%$ and $0.49 \% \pm 0.06 \%)$ and $\mathrm{CC}(0.15 \% \pm 0.03 \%$ and $0.12 \% \pm 0.02 \%$ ) concentrations in the dust and sediment samples were found, as compared to the particulate samples. A higher EC concentration in the studied area than in other locations reported in the literature, both in India and in the rest of world, was observed (Zong et al. 2016; Guha et al., 2015; Han et al. 2015; Ozdemir et al. 2014; Han et al. 2009). This may be tentatively ascribed to massive coal burning in the area, given that two of India's largest coal-fired power stations are operating in Chhattisgarh state.

The concentration of the individual PCBs congeners in the ambient air and particulates is presented in Table 1. The total concentration of PCBs in the ambient particulate matters of four locations ranged from 185.72 to $1,110.82 \mathrm{ng} \cdot \mathrm{g}^{-1}$, with an average value of $574.05 \pm 405.12 \mathrm{ng} \mathrm{g}^{-1}$. The concentrations of MCBs, DCBs, TCBs, TeCBs, PeCBs, HCBs, HeCBs, OCBs, and NCBs were the $137.01-720.4,4.82-10.33,6.62-87.39,4.66-129.47$, $17.13-78.57,7.78-45.87,0.41-6.55$, and $1.01-2.53 \mathrm{ng} \mathrm{g}^{-1}$ intervals, respectively, with the highest and the lowest values for MCBs $\left(364.82 \pm 267.29 \mathrm{ng} \mathrm{g}^{-1}\right)$ and NCBs $\left(1.74 \pm 0.62 \mathrm{ng} \mathrm{g}^{-1}\right)$, respectively. Similarly, the major portion of the PCBs in particulate samples was contributed by the MCBs congeners.

As regards PCBs in the road dust samples, 98 congeners were detected. Their concentrations in eight locations of highway are summarized in Table 2. The total concentration of PCBs ranged from 102 to $537 \mathrm{ng} \mathrm{g}^{-1}$, with an average value of $241 \mathrm{ng} \mathrm{g}^{-1}$. The concentrations of $\mathrm{MCB}_{k}$ DCBs, TCBs, TeCBs, PeCBs, $\mathrm{HCBs}$, HeCBs, OCBs, and NCBs were in the following intervals: 37.0-197.4, 7.2-32.4, 11.7-40.6, 0-170.5, 9.4-99.1, 15.4-29.8, $1.5-6.2,0.7-8.8$ and $0-1.3 \mathrm{ng} \mathrm{g}^{-1}$, respectively, with average values of $93.4 \pm 67.38,16.26 \pm 8.13,23.68 \pm 10.33,51.90 \pm 56.70$, $28.30 \pm 30.19,21.55 \pm 4.92,3.09 \pm 1.60,2.75 \pm 2.90$ and $0.16 \pm$ $0.46 \mathrm{ng} \mathrm{g}^{-1}$, respectively. The highest total concentration of PCBs was detected at the Khamtarai site, expected due to higher vehicular emissions. The concentration of the preminent congeners was detected in the following increasing order: NCBPs $<$ OCBPs $<$ HeCBPs $<$ DCBPs $<$ HCBPs $<$ TCBPs $<$ PCBPs $<$ TeCBPs $=$ MCBPs . The total PCBs concentration showed a fair correlation with the congener frequency $(r=0.61)$. It is worth noting that the concentration of PCBs $\left(241 \pm 146 \mathrm{ng} \mathrm{g}^{-1}\right)$ in the area of study was higher than those reported for Guangzhou, India; Hong Kong; Chennai, India; and Northern Vietnam (in the $0.25-228 \mathrm{ng} \mathrm{kg}^{-1}$ range) (Anh et al. 2019; Chakraborty et al. 2016; Wang et al. 2013).

In pond sediments, $84 \mathrm{PCBs}$ congeners were detected. Their concentrations in the 10 ponds are given in Table 3. The total concentration of PCBs ranged from 241 to $538 \mathrm{ng} \mathrm{g}^{-1}$, with a mean value of $328 \mathrm{ng} \mathrm{g}^{-1}$. The highest PCB content was found in the Raja pond, ascribed to increased human activities in the area. The concentrations of $\mathrm{MCB}_{k} \mathrm{DCB}_{2} \mathrm{TCB}_{k} \mathrm{TeCB}_{k}$ $\mathrm{PeCB}_{2} \mathrm{HCB}_{2} \mathrm{HeCB}_{2}$ and $\mathrm{OCB}_{1}$ were in the following ranges: 123.80-372.70, 7.30-29.90, 13.40-46.80, 5.90-74.50, 0-80.20, $0-4.10$, and $0-11.10 \mathrm{ng} \mathrm{g}^{-1}$, respectively, with mean values of $201.31 \pm 80.97,17.24 \pm 7.0,28.88 \pm 10.84,30.14 \pm 19.91,25.25$ $\pm 19.73,22.41 \pm 24.68,1.04 \pm 1.36$, and $1.31 \pm 3.47 \mathrm{ng} \mathrm{g}^{-1}$, respectively. A different trend of occurring ef $\mathrm{PCBs}$ congeners was found in this case: HeCBs $<$ OCBs $<$ DCBs $<$ HCBs $<$ PCBs $<$ $\mathrm{TCBs}<\mathrm{TeCBs}<\mathrm{MCBs}$. No NCBs were detected in the surface sediment. 
Table 2. Concentration of PCBs in road dust collected in $2008, \mathrm{ng} \mathrm{g}^{-1}$

\begin{tabular}{|c|c|c|c|c|c|c|c|c|c|c|c|}
\hline S. no. & Type & Congener & TB & HP & $\mathrm{S}$ & KT & $\mathrm{B}$ & $\mathrm{U}$ & SK & ST & Mean value \pm STD \\
\hline 1 & MCBs & 1 & 18.4 & 15.5 & 62.3 & 141.1 & 125.7 & 43.7 & 37.0 & 44.7 & $61.05 \pm 47.25$ \\
\hline 2 & & 3 & 18.6 & 23.7 & 43.3 & 52.7 & 71.7 & 35.0 & 0 & 13.8 & $32.35 \pm 23.13$ \\
\hline 3 & DCBs & $4+10$ & 0.0 & 2.7 & 0 & 1.6 & 0 & 0 & 0 & 0 & $0.54 \pm 1.04$ \\
\hline 4 & & 6 & 1.0 & 1.8 & 2.1 & 1.5 & 2.9 & 1.0 & 1.7 & 1.7 & $1.71 \pm 0.61$ \\
\hline 5 & & $7+9$ & 0.4 & 0.7 & 0.5 & 0.5 & 0.7 & 0.6 & 0.6 & 0.5 & $0.56 \pm 0.11$ \\
\hline 6 & & $8+5$ & 5.8 & 11.6 & 15.3 & 9.4 & 28.8 & 7.3 & 9.6 & 19.8 & $13.45 \pm 7.66$ \\
\hline 7 & TCBs & $16+32$ & 1.6 & 0.0 & 2.3 & 4.2 & 1.9 & 1.7 & 5.0 & 2.5 & $2.40 \pm 1.57$ \\
\hline 8 & & 17 & 7.0 & 8.7 & 5.3 & 5.1 & 1.9 & 1.8 & 1.8 & 4.8 & $4.55 \pm 2.57$ \\
\hline 9 & & 18 & 1.1 & 1.7 & 4.3 & 0.7 & 0.8 & 0.8 & 3.6 & 3.5 & $2.06 \pm 1.49$ \\
\hline 10 & & 19 & 0.5 & 1.8 & 1.4 & 1.1 & 1.9 & 0.5 & 0.9 & 0.9 & $1.13 \pm 0.54$ \\
\hline 11 & & $21+33+53$ & 0.0 & 0 & 1.6 & 4.6 & 1.2 & 1.3 & 0.8 & 5.9 & $1.93 \pm 2.16$ \\
\hline 12 & & 22 & 0.0 & 0 & 2.6 & 4.2 & 5.4 & 1.1 & 4.3 & 6.0 & $2.95 \pm 2.38$ \\
\hline 13 & & 24 & 1.1 & 0.9 & 1.7 & 0.8 & 1.1 & 0.5 & 1.9 & 0.9 & $1.11 \pm 0.47$ \\
\hline 14 & & 25 & 1.4 & 0 & 0.9 & 2.4 & 1.5 & 0.5 & 1.1 & 1.2 & $1.13 \pm 0.71$ \\
\hline 15 & & 26 & 0.4 & 0 & 0.6 & 1.7 & 1.3 & 0.6 & 0.9 & 1.4 & $0.86 \pm 0.57$ \\
\hline 16 & & $28+31$ & 1.3 & 0 & 4.5 & 6.7 & 4.0 & 2.3 & 2.4 & 12.9 & $4.26 \pm 4.05$ \\
\hline 17 & & 29 & 1.0 & 0 & 3.1 & 2.7 & 0.7 & 0.6 & 1.7 & 0.6 & $1.30 \pm 1.10$ \\
\hline 18 & TeCBs & $37+42$ & 0 & 0 & 11.2 & 14.4 & 4.9 & 1.6 & 2.0 & 22.4 & $7.06 \pm 8.16$ \\
\hline 19 & & 40 & 0 & 0 & 0.0 & 8.5 & 0 & 0 & 0 & 1.7 & $1.28 \pm 2.98$ \\
\hline 20 & & $41+64+71$ & 0 & 0 & 4.8 & 10.0 & 2.6 & 0.9 & 2.0 & 14.9 & $4.40 \pm 5.37$ \\
\hline 21 & & 44 & 0 & 0 & 2.0 & 9.5 & 2.1 & 0.4 & 0.9 & 3.0 & $2.24 \pm 3.13$ \\
\hline 22 & & 45 & 0 & 0 & 0.6 & 6.3 & 1.2 & 0.4 & 0.4 & 1.3 & $1.28 \pm 2.09$ \\
\hline 23 & & 46 & 0 & 0 & 7.9 & 14.9 & 6.9 & 1.1 & 3.0 & 1.6 & $4.43 \pm 5.18$ \\
\hline 24 & & $47+48$ & 0 & 0 & 1.0 & 9.4 & 1.0 & 0.7 & 0.0 & 1.9 & $1.75 \pm 3.16$ \\
\hline 25 & & 49 & 0 & 0 & 25.4 & 17.3 & 9.0 & 6.6 & 13.1 & 3.8 & $9.40 \pm 8.85$ \\
\hline 26 & & 51 & 0 & 0 & 0.7 & 2.4 & 0.0 & 0.5 & 0.0 & 0.0 & $0.45 \pm 0.83$ \\
\hline 27 & & $56+60$ & 0 & 0 & 2.9 & 5.3 & 5.5 & 2.5 & 2.9 & 6.4 & $3.19 \pm 2.42$ \\
\hline 28 & & 62 & 0 & 0 & 4.5 & 7.1 & 2.0 & 0.9 & 1.3 & 3.7 & $2.44 \pm 2.49$ \\
\hline 29 & & 63 & 0 & 0 & 0.6 & 3.3 & 0.8 & 0.0 & 0.0 & 0.8 & $0.69 \pm 1.12$ \\
\hline 30 & & $66+95$ & 0 & 0 & 1.0 & 21.8 & 0.7 & 1.2 & 0.0 & 4.3 & $3.63 \pm 7.48$ \\
\hline 31 & & 70.76 & 0 & 0 & 10.3 & 33.3 & 4.6 & 1.2 & 2.8 & 8.4 & $7.58 \pm 1.06$ \\
\hline 32 & & 74 & 0 & 0 & 2.0 & 7.0 & 0.7 & 0.6 & 0.7 & 5.9 & $2.11 \pm 2.76$ \\
\hline 33 & $\mathrm{PeCBs}$ & $77+110$ & 6.5 & 0.8 & 6.4 & 1.8 & 4.4 & 1.8 & 2.6 & 1.4 & $3.21 \pm 2.26$ \\
\hline 34 & & $81+87$ & 0 & 0 & 0 & 1.1 & 0.8 & 0.4 & 0.7 & 0.2 & $0.40 \pm 0.42$ \\
\hline 35 & & $82+151$ & 1.0 & 3.8 & 0 & 0.0 & 0.0 & 0.8 & 1.2 & 0.0 & $0.85 \pm 1.30$ \\
\hline 36 & & 83 & 0.4 & 0.4 & 1.7 & 1.2 & 0.8 & 0.4 & 0.5 & 0.7 & $0.76 \pm 0.47$ \\
\hline 37 & & 85 & 0 & 0 & 0 & 0.7 & 0.5 & 12.8 & 0.5 & 0.1 & $1.83 \pm 4.44$ \\
\hline 38 & & 89 & 0.4 & 3.1 & 1.2 & 2.8 & 3.5 & 0.9 & 2.1 & 1.4 & $1.93 \pm 1.12$ \\
\hline 39 & & 91 & 0.0 & 0.0 & 9.9 & 82.5 & 0.0 & 15.4 & 0.0 & 0.0 & $13.48 \pm 28.51$ \\
\hline 40 & & 97 & 0.1 & 0.2 & 0.3 & 1.0 & 0.9 & 0.3 & 0.7 & 0.7 & $0.53 \pm 0.34$ \\
\hline 41 & & 99 & 0.0 & 0.1 & 0.2 & 0.2 & 0.1 & 0.8 & 0.1 & 0.1 & $0.20 \pm 0.25$ \\
\hline 42 & & 100 & 0.0 & 0.0 & 0.5 & 4.1 & 1.5 & 0.4 & 0.6 & 1.9 & $1.13 \pm 1.38$ \\
\hline 43 & & 101 & 0.7 & 1.0 & 1.1 & 1.3 & 1.6 & 0.8 & 0.9 & 0.1 & $0.94 \pm 0.44$ \\
\hline 44 & & 105 & 1.1 & 1.3 & 1.0 & 1.1 & 1.3 & 1.7 & 1.5 & 1.4 & $1.30 \pm 0.23$ \\
\hline 45 & & 118 & 0.7 & 0.5 & 0 & 0.4 & 0 & 0.8 & 0.8 & 0.5 & $0.46 \pm 0.32$ \\
\hline 46 & & $123+149$ & 1.5 & 1.4 & 0.9 & 0.9 & 0.8 & 2.4 & 1.6 & 0.9 & $1.30 \pm 0.55$ \\
\hline 47 & $\mathrm{HCBs}$ & $129+178$ & 0 & 0 & 0.7 & 1.2 & 0 & 0.4 & 1.3 & 1.2 & $0.60 \pm 0.58$ \\
\hline 48 & & 132 & 0.7 & 0.3 & 0.6 & 0.5 & 0.9 & 0.8 & 1.1 & 0.8 & $0.71 \pm 0.25$ \\
\hline 49 & & 134 & 0 & 3.2 & 0.0 & 0.7 & 0.0 & 1.3 & 1.4 & 0.6 & $0.90 \pm 1.09$ \\
\hline 50 & & $135+144$ & 1.1 & 2.6 & 1.5 & 2.4 & 0.0 & 2.0 & 3.1 & 1.7 & $1.80 \pm 0.97$ \\
\hline 51 & & 136 & 0.3 & 0.8 & 0 & 1.3 & 1.3 & 0.9 & 1.9 & 0.3 & $0.85 \pm 0.64$ \\
\hline 52 & & $137+130+176$ & 0 & 0 & 0 & 0.6 & 0.8 & 0.4 & 0.9 & 0.6 & $0.41 \pm 0.37$ \\
\hline 53 & & $138+158$ & 19.2 & 1.0 & 0 & 0.0 & 14.2 & 0.0 & 0.0 & 0.0 & $4.30 \pm 7.78$ \\
\hline 54 & & 141 & 0.6 & 0.8 & 0.8 & 1.1 & 1.3 & 0.7 & 1.3 & 1.0 & $0.95 \pm 0.27$ \\
\hline 55 & & 146 & 1.1 & 0 & 0 & 1.0 & 1.3 & 1.2 & 1.7 & 0.9 & $0.90 \pm 0.60$ \\
\hline 56 & & 153 & 2.3 & 3.6 & 2.2 & 4.3 & 4.0 & 3.5 & 0.0 & 3.3 & $2.90 \pm 1.39$ \\
\hline 57 & & 157 & 0.5 & 2.3 & 0.9 & 1.2 & 0.0 & 0.0 & 0.0 & 1.6 & $0.81 \pm 0.85$ \\
\hline 58 & & 163 & 0.0 & 8.2 & 23.1 & 5.4 & 0.0 & 4.2 & 3.6 & 6.8 & $6.41 \pm 7.35$ \\
\hline 59 & $\mathrm{HeCBs}$ & $170+190$ & 0 & 0 & 0 & 0 & 0 & 0 & 0 & 2.0 & $0.25 \pm 0.71$ \\
\hline 60 & & 177 & 1.1 & 0.5 & 1.3 & 1.7 & 1.4 & 0.5 & 1.3 & 1.6 & $1.18 \pm 0.46$ \\
\hline 61 & & 183 & 0.6 & 0.0 & 0.5 & 1.0 & 0.9 & 0.5 & 1.3 & 1.1 & $0.74 \pm 0.42$ \\
\hline 62 & & 185 & 0.2 & 0.0 & 0.1 & 1.8 & 1.3 & 0.5 & 0.2 & 1.5 & $0.70 \pm 0.72$ \\
\hline 63 & & $187+182$ & 0 & 1.8 & 0 & 0 & 0 & 0 & 0 & 0 & $0.23 \pm 0.64$ \\
\hline
\end{tabular}


Table 2. (Continued.)

\begin{tabular}{|c|c|c|c|c|c|c|c|c|c|c|c|}
\hline S. no. & Type & Congener & TB & $\mathrm{HP}$ & $\mathrm{S}$ & KT & B & $\mathrm{U}$ & SK & ST & Mean value \pm STD \\
\hline 64 & OCBs & 194 & 0 & 0 & 0 & 0 & 0 & 0 & 0 & 0.5 & $0.06 \pm 0.18$ \\
\hline 65 & & $196+203$ & 0 & 2.8 & 0 & 0 & 0 & 0 & 0 & 1.4 & $0.53 \pm 1.04$ \\
\hline 66 & & 198 & 0 & 2.2 & 0 & 0 & 0 & 0 & 0 & 1.0 & $0.40 \pm 0.81$ \\
\hline 67 & & 201 & 2.1 & 3.8 & 1.1 & 1.4 & 1.2 & 0.7 & 1.1 & 2.7 & $1.76 \pm 1.04$ \\
\hline 68 & $\mathrm{NCBs}$ & $208+195$ & 0 & 0 & 0 & 0 & 0 & 0 & 0 & 1.3 & $0.16 \pm 0.46$ \\
\hline
\end{tabular}

Note: $\mathrm{TB}=$ Tatibandh; $\mathrm{HP}=$ Hirapur; $\mathrm{SA}=\mathrm{S} ; \mathrm{KT}=$ Khamtarai; $\mathrm{B}=$ Birgaon; $\mathrm{U}=$ Urla; $\mathrm{SK}=$ Sankra; and $\mathrm{ST}=$ Siltara.

When the PCBs concentration in the pond sediment samples was analyzed as a function of depth, it was observed that the concentrations of MCBs, DCBs, TCBs, TeCBs, PeCBs, and HeCBs congeners increased steeply with depth, probably due to poor adsorption on the sediment particles (Fig. 2). However, the concentration of $\mathrm{HCB}_{\perp}$ decreased as the depth increased, suggesting an adsorption by the top-layer sedimentary particles (Fig. 2). A noticeable vertical distribution of congeners $1,3,4+10,6,7+9,8+5$, $17,18,19,77+110$, and 85 was observed.

For comparison purposes, the PCBs concentration in six ponds in Bhilai and Korba varied from 201 to $648 \mathrm{ng} \cdot \mathrm{g}^{-1}$ and from 404 to $773 \mathrm{ng} \mathrm{g}^{-1}$, with average values of $480 \pm 150$ and $561 \pm$ $155 \mathrm{ng} \mathrm{g}^{-1}$, respectively (Patel et al. 2013). The concentrations of aforementioned congeners in these two cities (in which huge quantities of coal are burnt for steel and electricity production) were even higher than those found in Raipur. It is also worth noting that the concentration of PCBs $\left(328 \pm 99 \mathrm{ng} \mathrm{g}^{-1}\right)$ in the pond sediments from the area of study was higher than those observed $\left(<0.01-126.49 \mathrm{ng} \mathrm{g}^{-1}\right)$ in soil/sediment of other locations (Jin et al. 2012; Li et al. 2012; Kumar et al. 2011), except for Harbor Island East, with concentrations of up to at $1,387 \mathrm{ng} \mathrm{g}^{-1}$ (Neira et al. 2018).

\section{Comparison of PCBs Concentrations in Environmental Samples}

Comparable total PCBs concentrations were observed in the pond sediments and road dust samples, while their concentration in the particulate samples was markedly increased [Fig. 3(a)]. The highest concentrations of MCBs, HeCBs, OCBs, and NCBs were found in the particulates; those of $\mathrm{TeCBs}$ and $\mathrm{PeCBs}$ in the road dusts (Fig. 3); and those of DCBs, TCBs, and HCBs in the sediments (Fig. 3). Remarkably higher contents of MCBs and HeCBs were detected in the PM samples, which could be an indicator of air pollution.

A high concentration of congeners $3,17,89$, and $138+158$ was identified in the PM and sediment samples, indicating emissions multiple sources. The dominant concentration of congeners $1,8+$ 5, 49, and 91 was registered in the road dust samples, emissions mainly vehicles.

As per the ANOVA test, the uncertainty $(F)$ value for the PCBs concentration in the particulates, road dust, and sediments were found to be 7, 65, 535, and 29, indicating multiple emission sources of the PCBs in the road geo-media.

\section{Temporal Evolution of PCBs Concentration}

The total concentrations of PCBs in particulate matter (Kota), road dust (Khamtarai), and sediments (Raja pond) were monitored over an eight-year period (2008-2015), as shown in Fig. 4, and registered a gradual increase from 428 to $670 \mathrm{pg} \mathrm{m}^{-3}$, from 537 to 752 and from 538 to $775 \mathrm{ng} \mathrm{g}^{-1}$, with respectively, probably due to increased vehicular and industrial emissions (Fig. 4). These data represented an average annual increment rate of $6.2 \% \pm 3.2 \%, 4.9 \% \pm 2.1 \%$, and $5.4 \% \pm 1.1 \%$ in PCBs concentrations, respectively.

\section{Toxic Equivalency Factor of PCBs}

The toxic equivalency factor (TEF) denotes the toxicity of individual congeners in terms of the most toxic form of dioxin: 2,3,7,8-tetrachlorodibenzo-p-dioxin (2,3,7,8-TCDD), for which a value of 1.0 is assigned (USEPA 2010). Among the congeners found, PCB-126 (3,3',4,4',5-pentachlorobiphenyl) was the most toxic (USEPA 2010), with a TEF of 0.1 , while the TEF factors for PCB-77 (3,3',4,4'-tetrachlorobiphenyl), PCB-81 $\left(3,4,4^{\prime}, 5\right.$-tetrachlorobiphenyl), and others are $0.0001,0.0003$, and 0.00003 , respectively. Among the aforementioned "dioxin-like" congeners, $77,81,105,118,123$, and 157 were detected in most of the particulate matter, road dust, and sediment samples. The normalized total mean TEFs for the PM, road dust, and sediments from Raipur city were estimated at $0.00066,0.00058$, and 0.00047 , respectively.

\section{Correlations and Sources}

Several chemical species, including EC, OC, polycyclic aromatic hydrocarbons (PAHs), and biphenyls (BPs), are emitted during the combustion processes of fuels (Laroo et al. 2012; Hesterberg et al. 2008; $\mathrm{Na}$ et al. 2004).

The main PCBs emission sources are the chlorination of biphenyls during the combustion process of fuels and other materials, vaporization; leakage from application sites of Aroclors; and burning, disposal, and dumping of PCBs-containing materials (Meijer et al. 2003).

The combustion of fuels, industrial/metallurgical activities and power generation processes have been reported as possible sources of PCBs, due to reaction of carbon and chlorine at the combustion source (Biterna and Voutsa 2005; Dyke et al. 2003; Weber et al. 2001).

The total PCBs content showed a fair correlation (at $r=0.73$ 0.75 ) with PM, EC, OC, and CC, indicating that PCBs would be partly originated from burning processes (Table 4). MCBs, DCBs, and TCBs had a good correlation with TCBs, TeCBs, $\mathrm{HeCBs}$, and OCBs $(r=0.79-0.93)$; TeCBs, PeCBs, HCBs, HeCBs, and NCBs $(r=0.76-0.91)$; and MCBs, HeCBs, and OCBs $(r=0.91-0.96)$, respectively, indicating that they would be originated by the chlorination process of lower congeners $(1 b)-(1 g)$ as follows (Biterna and Voutsa 2005; Dyke et al. 2003; Weber et al. 2001)

$$
\begin{gathered}
\mathrm{C}_{12} \mathrm{H}_{10}+\mathrm{Cl}_{2} \rightarrow \mathrm{C}_{12} \mathrm{H}_{9} \mathrm{Cl}+\mathrm{HCl} \\
\mathrm{C}_{12} \mathrm{H}_{9} \mathrm{Cl}+\mathrm{Cl}_{2} \rightarrow \mathrm{C}_{12} \mathrm{H}_{8} \mathrm{Cl}_{2}+\mathrm{HCl} \\
\mathrm{C}_{12} \mathrm{H}_{8} \mathrm{Cl}_{2}+\mathrm{Cl}_{2} \rightarrow \mathrm{C}_{12} \mathrm{H}_{7} \mathrm{Cl}_{3}+\mathrm{HCl}
\end{gathered}
$$


Table 3. Distribution of PCBs in sediments of Raipur city collected in $2008, \mathrm{ng} \mathrm{g}^{-1}$

\begin{tabular}{|c|c|c|c|c|c|c|c|c|c|c|c|c|c|}
\hline S. no. & Type & Congener & $\mathrm{S} 1$ & $\mathrm{~S} 2$ & $\mathrm{~S} 3$ & S4 & S5 & S6 & S7 & S8 & S9 & $\mathrm{S} 10$ & Mean value \pm STD \\
\hline 1 & MCBs & 1 & 66.9 & 68.7 & 79.2 & 103.5 & 64.6 & 81.0 & 116.7 & 89.3 & 124.3 & 95.2 & $88.94 \pm 20.86$ \\
\hline 2 & & 3 & 65.8 & 165.1 & 44.6 & 54.5 & 87.6 & 58.7 & 93.7 & 283.4 & 174.9 & 95.4 & $112.37 \pm 74.67$ \\
\hline 3 & $\mathrm{DCBs}$ & $4+10$ & 0 & 4.8 & 0 & 0 & 5.7 & 0.0 & 4.1 & 5.9 & 10.7 & 0 & $3.12 \pm 3.72$ \\
\hline 4 & & 6 & 2.4 & 0.1 & 1.6 & 2.0 & 1.5 & 2.4 & 4.7 & 4.7 & 4.8 & 3.1 & $2.73 \pm 1.59$ \\
\hline 5 & & $7+9$ & 0.7 & 0.0 & 0.6 & 0.7 & 0.8 & 0.8 & 0.4 & 2.1 & 1.7 & 0.7 & $0.85 \pm 0.61$ \\
\hline 6 & & $8+5$ & 17.1 & 2.4 & 14.9 & 10.2 & 4.0 & 6.4 & 12.8 & 11.3 & 12.7 & 13.6 & $10.54 \pm 4.81$ \\
\hline 7 & TCBs & $16+32$ & 2.0 & 2.4 & 5.2 & 4.6 & 4.0 & 2.3 & 4.3 & 3.1 & 4.4 & 2.6 & $3.49 \pm 1.14$ \\
\hline 8 & & 17 & 0 & 0 & 4.3 & 7.2 & 5.8 & 3.6 & 4.4 & 4.3 & 7.0 & 9.5 & $4.61 \pm 3.01$ \\
\hline 9 & & 18 & 1.8 & 0.1 & 2.9 & 2.7 & 2.5 & 1.8 & 1.3 & 1.5 & 2.4 & 1.4 & $1.84 \pm 0.83$ \\
\hline 10 & & 19 & 2.0 & 0.3 & 1.7 & 2.4 & 3.4 & 3.5 & 2.6 & 1.4 & 6.8 & 2.8 & $2.69 \pm 1.73$ \\
\hline 11 & & $21+33+53$ & 2.2 & 1.3 & 2.5 & 2.0 & 4.6 & 1.9 & 1.2 & 1.7 & 4.2 & 1.6 & $2.32 \pm 1.17$ \\
\hline 12 & & 22 & 2.8 & 1.9 & 3.2 & 2.4 & 8.7 & 2.0 & 1.8 & 2.2 & 4.6 & 0.7 & $3.03 \pm 2.24$ \\
\hline 13 & & 24 & 0.0 & 0.8 & 1.0 & 1.3 & 1.3 & 0.9 & 0 & 0 & 1.7 & 1.1 & $0.81 \pm 0.61$ \\
\hline 14 & & 25 & 1.7 & 1.0 & 2.0 & 2.1 & 2.7 & 1.2 & 1.6 & 1.5 & 2.3 & 0.9 & $1.70 \pm 0.58$ \\
\hline 15 & & 26 & 0.9 & 1.6 & 2.1 & 2.0 & 5.2 & 3.0 & 1.4 & 1.5 & 4.9 & 0.7 & $2.33 \pm 1.57$ \\
\hline 16 & & $28+31$ & 4.1 & 3.2 & 5.5 & 4.8 & 6.4 & 3.5 & 3.8 & 3.7 & 5.6 & 3.3 & $4.39 \pm 1.12$ \\
\hline 17 & & 29 & 1.9 & 0.8 & 2.4 & 2.6 & 2.2 & 1.9 & 1.5 & 1.8 & 1.5 & 0.1 & $1.67 \pm 0.75$ \\
\hline 18 & TeCBs & $37+42$ & 2.0 & 1.9 & 3.2 & 0.9 & 10.4 & 2.0 & 1.6 & 0.0 & 6.5 & 2.2 & $3.07 \pm 3.10$ \\
\hline 19 & & $41+64+71$ & 2.7 & 1.5 & 12.4 & 1.4 & 5.1 & 1.2 & 1.6 & 1.2 & 6.8 & 0.9 & $3.48 \pm 3.69$ \\
\hline 20 & & 44 & 1.0 & 0.8 & 0.6 & 0.8 & 4.6 & 0.7 & 0.7 & 0.7 & 3.6 & 0.4 & $1.39 \pm 1.46$ \\
\hline 21 & & 45 & 1.1 & 0.8 & 1.2 & 2.0 & 4.2 & 0.6 & 0.8 & 0.7 & 3.0 & 0.6 & $1.50 \pm 1.21$ \\
\hline 22 & & 46 & 1.1 & 0.7 & 1.3 & 1.1 & 3.8 & 0 & 1.7 & 1.0 & 2.6 & 0.8 & $1.41 \pm 1.08$ \\
\hline 23 & & $47+48$ & 1.6 & 0 & 1.1 & 0 & 3.9 & 0 & 1.8 & 0 & 2.8 & 0.8 & $1.20 \pm 1.35$ \\
\hline 24 & & 49 & 2.7 & 1.9 & 3.2 & 4.0 & 4.8 & 0 & 1.9 & 1,4 & 3.4 & 2.7 & $2.60 \pm 1.37$ \\
\hline 25 & & 51 & 2.4 & 0 & 2.2 & 0 & 4.7 & 1.9 & 0.0 & 0 & 2.3 & 0.0 & $1.35 \pm 1.61$ \\
\hline 26 & & $56+60$ & 4.0 & 4.4 & 4.2 & 0 & 10.4 & 3.5 & 4.4 & 0 & 8.0 & 8.6 & $4.75 \pm 3.42$ \\
\hline 27 & & 62 & 1.7 & 1.2 & 1.6 & 1.4 & 4.3 & 0 & 1.8 & 1.2 & 3.3 & 1.5 & $1.80 \pm 1.19$ \\
\hline 28 & & 63 & 1.2 & 0 & 1.0 & 0 & 1.5 & 1.1 & 0 & 0 & 2.4 & 0 & $0.72 \pm 0.85$ \\
\hline 29 & & $66+95$ & 2.7 & 1.5 & 2.2 & 1.8 & 5.0 & 3.4 & 1.6 & 1.1 & 4.7 & 1.4 & $2.54 \pm 1.39$ \\
\hline 30 & & 70.76 & 3.1 & 1.8 & 2.5 & 1.8 & 5.7 & 2.3 & 2.1 & 1.5 & 4.1 & 2.5 & $2.74 \pm 1.28$ \\
\hline 31 & & 74 & 2.0 & 1.0 & 1.2 & 0.9 & 3.3 & 1.3 & 1.0 & 0.8 & 3.5 & 0.9 & $1.59 \pm 1.01$ \\
\hline 32 & $\mathrm{PeCBs}$ & $77+110$ & 5.8 & 0.4 & 1.8 & 1.1 & 3.2 & 2.3 & 2.2 & 1.0 & 7.2 & 4.1 & $2.91 \pm 2.20$ \\
\hline 33 & & $81+87$ & 0.8 & 0 & 0 & 0 & 1.2 & 0.6 & 0 & 0 & 0 & 0.4 & $0.30 \pm 0.43$ \\
\hline 34 & & $82+151$ & 2.3 & 0 & 0 & 0 & 2.8 & 0 & 0 & 0 & 0 & 5.1 & $1.02 \pm 1.79$ \\
\hline 35 & & 83 & 0.9 & 0 & 0.1 & 0.5 & 0.7 & 1.1 & 0.3 & 0.5 & 1.0 & 1.5 & $0.66 \pm 0.47$ \\
\hline 36 & & 85 & 8.2 & 0 & 7.5 & 4.1 & 3.8 & 15.3 & 0.1 & 11.4 & 0 & 0.3 & $5.07 \pm 5.39$ \\
\hline 37 & & 89 & 2.4 & 0.9 & 0 & 1.1 & 6.5 & 3.5 & 1.1 & 0.1 & 3.9 & 2.3 & $2.18 \pm 2.01$ \\
\hline 38 & & 91 & 0 & 15.0 & 0 & 0 & 46.8 & 0 & 0 & 0 & 0 & 0 & $6.18 \pm 15.03$ \\
\hline 39 & & 97 & 0.6 & 0.1 & 0.1 & 0.3 & 1.3 & 0.4 & 0.2 & 0.2 & 0.2 & 0.9 & $0.43 \pm 0.39$ \\
\hline 40 & & 99 & 0.8 & 0.7 & 0.3 & 1.3 & 1.1 & 1.0 & 0.5 & 0.7 & 1.4 & 0.6 & $0.84 \pm 0.35$ \\
\hline 41 & & 100 & 1.6 & 0.7 & 1.7 & 1.4 & 2.0 & 2.3 & 0.5 & 0.8 & 1.1 & 0.9 & $1.30 \pm 0.60$ \\
\hline 42 & & 101 & 2.0 & 1.3 & 0.7 & 1.7 & 2.9 & 1.6 & 1.0 & 0.4 & 3.4 & 1.9 & $1.69 \pm 0.93$ \\
\hline 43 & & 105 & 4.3 & 0 & 0 & 0 & 2.2 & 1.6 & 0 & 0 & 3.1 & 2.1 & $1.33 \pm 1.57$ \\
\hline 44 & & 118 & 3.5 & 0 & 0 & 0 & 0 & 0 & 0 & 0 & 0 & 0 & $0.35 \pm 1.11$ \\
\hline 45 & & $123+149$ & 5.9 & 0 & 1.1 & 0 & 0 & 0 & 0 & 0 & 0 & 2.9 & $0.99 \pm 1.96$ \\
\hline 46 & $\mathrm{HCBs}$ & $129+178$ & 1.8 & 0 & 0 & 0 & 1.4 & 0.7 & 0 & 0 & 3.3 & 0 & $0.72 \pm 1.13$ \\
\hline 47 & & 132 & 2.6 & 0 & 0 & 0 & 0.8 & 0.7 & 0.9 & 0 & 0 & 1.6 & $0.66 \pm 0.88$ \\
\hline 48 & & 134 & 8.3 & 0 & 0 & 0 & 0 & 0 & 0 & 0 & 0 & 3.3 & $1.16 \pm 2.71$ \\
\hline 49 & & $135+144$ & 2.1 & 0 & 1.4 & 0.7 & 3.5 & 0.8 & 1.4 & 0 & 1.8 & 2.9 & $1.46 \pm 1.16$ \\
\hline 50 & & 136 & 4.4 & 0 & 0 & 0 & 3.8 & 0.9 & 0 & 0 & 0 & 2.1 & $1.12 \pm 1.72$ \\
\hline 51 & & $137+130+176$ & 2.1 & 0 & 0 & 0 & 0 & 0 & 0 & 0 & 2.0 & 0 & $0.41 \pm 0.86$ \\
\hline 52 & & $138+158$ & 13.6 & 0 & 5.6 & 5.5 & 6.4 & 14.6 & 7.8 & 5.6 & 71.2 & 12.6 & $14.29 \pm 20.49$ \\
\hline 53 & & 141 & 2.2 & 0 & 0.3 & 0 & 1.3 & 0.9 & 0.6 & 0 & 1.9 & 0 & $0.72 \pm 0.83$ \\
\hline 54 & & 146 & 2.3 & 0 & 0 & 0 & 0 & 0 & 0 & 0 & 0 & 0 & $0.23 \pm 0.73$ \\
\hline 55 & & 153 & 7.8 & 0 & 0 & 0 & 0 & 0 & 0 & 0 & 0 & 5.5 & $1.33 \pm 2.86$ \\
\hline 56 & & 157 & 1.7 & 0 & 0 & 1.4 & 0 & 0 & 0 & 0 & 0 & 0 & $0.31 \pm 0.66$ \\
\hline 57 & $\mathrm{HeCBs}$ & 177 & 0.0 & 0 & 0 & 0 & 0.7 & 0 & 0 & 0 & 1.8 & 0 & $0.25 \pm 0.59$ \\
\hline 58 & & 183 & 1.2 & 0 & 0 & 0 & 0 & 0 & 0 & 0 & 1.6 & 1.0 & $0.38 \pm 0.63$ \\
\hline 59 & & 185 & 0.8 & 0 & 0 & 0.7 & 1.2 & 0 & 0.1 & 0 & 0.7 & 0.6 & $0.41 \pm 0.44$ \\
\hline 60 & $\mathrm{OCBs}$ & 194 & 0.8 & 0 & 2.8 & 0 & 0 & 0 & 0 & 0 & 1.2 & 0 & $0.48 \pm 0.92$ \\
\hline 61 & & $208+195$ & 0.0 & 0 & 8.3 & 0 & 0 & 0 & 0 & 0 & 0 & 0 & $0.83 \pm 2.62$ \\
\hline
\end{tabular}

Note: S1 = Siltara; S2 = Urkura; S3 = Birgoan; S4 = Sarora; S5 = Rohnipuram; S6 = Ashi; S7 = Budheshwar; S8 = Vivekanand; S9= Raja; and S10 = Pandri. 

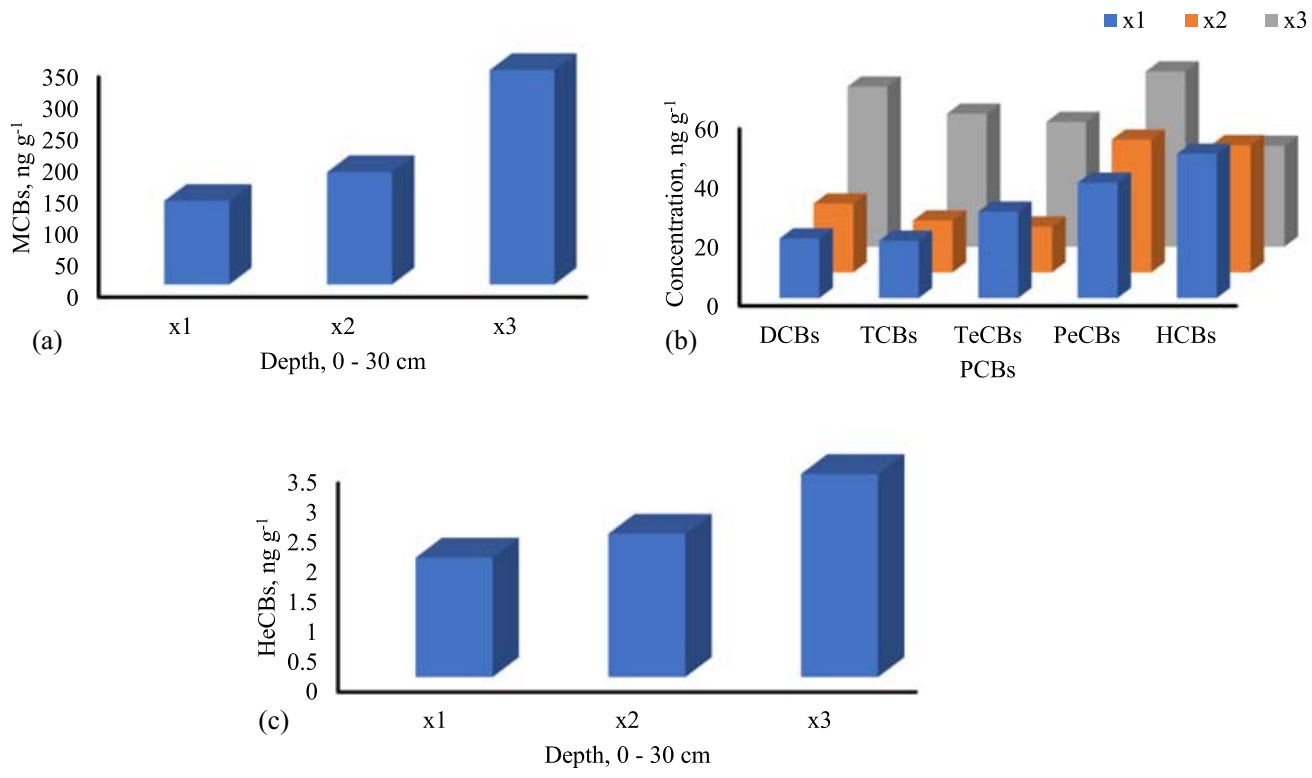

Fig. 2. Vertical concentration variation of PCBs in Siltara sediment. $x 1=0-10 \mathrm{~cm}, x 2=10-20 \mathrm{~cm}, x 3=20-30 \mathrm{~cm}$ : (a) monochlobiphenyls (MCBs); (b) mono-, di-, tri-, tetra-, penta-, and hexachlorobiphenyls (MCBs, DCBs, TCBs, TeCBs, PCBs, and HCBs); and (c) heptachlorobiphenyls (HeCBs).
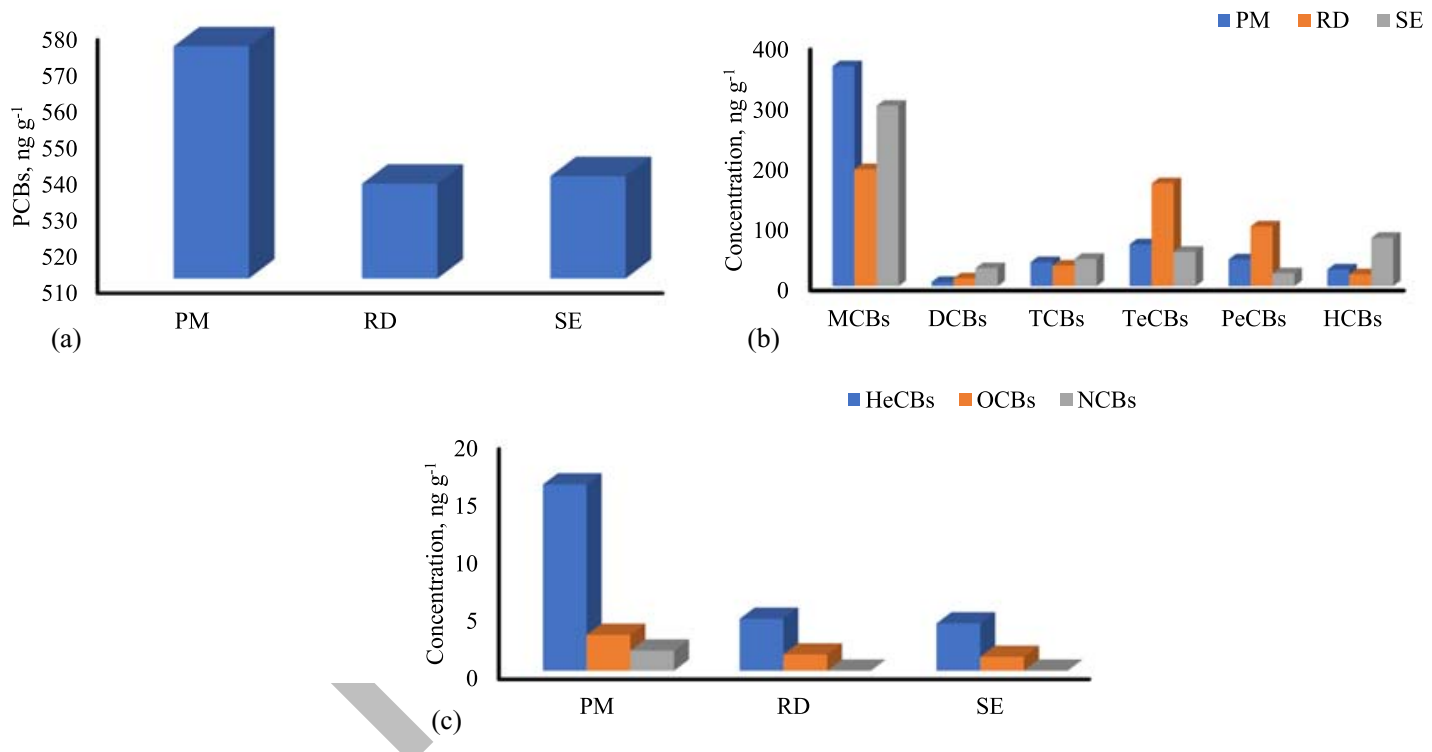

Fig. 3. PCBs concentration variation in particulate matter (PM), road dust (RD), and sediment (SE): (a) polychlorobiphenyls (PCBPs); (b) di-, tri-, tetra-, penta- and hexachlorobiphenyls (DCBs, TCBs, TeCBs, PCBs and HCBs); and (c) hepta-, octa-, and nonachlorobiphenyls (HeCBs, OCBs, and NCBs).

$$
\begin{aligned}
2 \mathrm{C}_{12} \mathrm{H}_{7} \mathrm{Cl}_{3}+2 \mathrm{Cl}_{2} & \rightarrow 2 \mathrm{C}_{12} \mathrm{H}_{6} \mathrm{Cl}_{4}+2 \mathrm{HCl} \\
2 \mathrm{C}_{12} \mathrm{H}_{6} \mathrm{Cl}_{4}+2 \mathrm{Cl}_{2} & \rightarrow 2 \mathrm{C}_{12} \mathrm{H}_{5} \mathrm{Cl}_{5}+2 \mathrm{HCl} \\
2 \mathrm{C}_{12} \mathrm{H}_{5} \mathrm{Cl}_{5}+2 \mathrm{Cl}_{2} & \rightarrow 2 \mathrm{C}_{12} \mathrm{H}_{4} \mathrm{Cl}_{6}+2 \mathrm{HCl}
\end{aligned}
$$

The Varimax rotation method, a commonly used orthogonal rotation method, was selected during the analysis. If the numbers of the both compounds and samples in the data set less than $50 \%$ of the total data, these compounds and samples were excluded from the analysis. Furthermore, prior to the analysis, the values lower the minimum detection limit (MDL) were replaced with half of MDL (Shyu et al. 2011).
For the PCBs emission in the ambient air, three factors were extracted out (Table S1). Factor-I contributed $49.762 \%$ of the total variance and was correlated with congeners $8+5,21+33+53$, $77+110,82+151,85,105,118,132,134,136,137+130+$ $176,138+158,141,153,178+129,180+193,196+203$, and 198 , identified as anthropogenic sources associated with the use of commercial Aroclor mixtures (Ikonomou et al. 2002). Factor-II, which accounted for $26.658 \%$ of the total variance, was highly correlated with congeners $1,28+31,56+60,89,101,174,177,183$, $185,202+171+156,201$, and 206 , which mainly originate from coal and wood combustion (Dumanoglu et al. 2017). Furthermore, most higher chlorinated congeners 151, 135, 144, 141, 179, 182, $187,183,174,181,177,180,193,170,190,199,203,196$, and 194, are also accepted to correlate with Aroclor 1260 (Jin et al. 
2012). Therefore, Factor-II would represent mixed sources that originate both from wood and coal combustion and Aroclor 1260 uses. Factor-III accounted for $23.98 \%$ of the total variance, related to congeners $81+87,44,47+48$, and 62 , which are associated with combustion and industrial thermal processes (Ikonomou et al. 2002; Mao et al. 2019).

In the case of road dust samples (Table S1), Factor-I eontribut33.388\% of the total variance and was strongly loaded with congeners $21+33+51,25,26,28+31,37+42,41+64+71$, $44,45,46,47+48,56+60,62,63,66+95,70+76,74,97$, 100, and 101. The main ones, 49, 52, 28, 44, 101, 110, and 118, are emitted from wood and coal combustions (Dumanoglu et al. 2017; Lee et al. 2005). Factor-II (17.616\%) mostly correlated with higher congeners: $16+32,22,82+151,132,136,137+$ $130+176,141,146,153,177,183$, and 185, some of which are the predominant components of Aroclor mixtures-1260 and 1254 (Du et al. 2008). Factor-III (14.452\%) was strongly loaded with congeners $1,3,6,7+9,8+5,17,19$, and 89. Congeners 10, 4, 15, and 17 are associated with Aroclor-1248 (Jin et al. 2012). The last factor, which explained $13.103 \%$ of the total variance, was found to be highly loaded with congeners: $18,24,29,49,77$ $+110,83$, and 163 . The planar congener 77 contamination to ambient attributable is likely due to commercial formulations (Tanabe et al. 1987). Therefore, the pattern of the road dust data was found to be close to emissions originated from the Aroclor mixtures that have been widely used in electrical transformers, dielectric fluids, heat transfer and hydraulic systems, paints, polymers, lubricants, plasticizers, fire retardants, immersion oils, sealants, and caulking compounds (Kodavanti et al. 2017; Anh et al. 2019).

For the pond sediment contaminations (Table S1), Factor-I accounted for $42.499 \%$ of the total variance, and was composed of

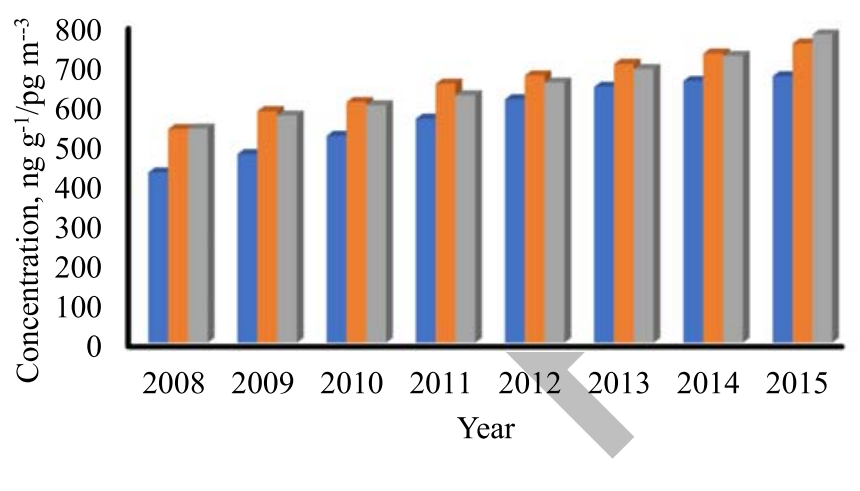

$\square \mathrm{PM} \square \mathrm{RD} \square \mathrm{SE}$

Fig. 4. Temporal variation of $\mathrm{PCBs}$ concentration in the particulate matters (PM), road dust (RD), and sediment (SE). congeners $28+31,29,41+64+71,44,46,49,62,66+95,70$ $+76,83$, and 101 . Hence, it would be related to combustion processes, as congeners 49, 28, 44, and 101 are mainly originated from coal and wood combustion (Dumanoglu et al. 2017). Factor-II, which explained $19.628 \%$ of the data variance, included congeners $8+5,16+32,21+33+53,24,26,37+42,44,45$, and $47+48$. The engeners $44,47,51$, and 68 are a tracer of the combustion and industrial thermal process. Especially, municipal waste incinerators have an important effect on the emissions of these congeners in the atmosphere (Ikonomou et al. 2002; Mao et al. 2019). Therefore, Factor-II was associated with the-industrial thermal process. Factor-III explained $12.531 \%$ of the total variance, and was loaded with higher chlorinated PCBs, including 1, 6, 7+9, 18, $74,135+144,138+158$, and 141. Higher chlorinated PCBs, $135,141,144$, and 138, mostly originate from Aroclor 1260 (Jin et al. 2012). Therefore, the contribution of this factor may be attributed to the use of Aroclor 1260.

\section{Comparison with PCBs Concentrations Reported for Other Regions}

A comparison with PCBs environmental contamination in various sites around the world is presented in Table 5. Total PCBs concentrations in the ambient air and particulate phase reported for Atlantic Ocean, India coastal area, East Africa, Turkey, France, Pohang (Korea), Karachi and Lahore, Panjab (Pakistan), Kanpur (India), London, and Yokohama (Japan) were found to be in the range: $4-220,216-1,077,65.6-244 \mathrm{pg} \mathrm{m}^{-3}, 52-293 \mathrm{ng} \mathrm{m}^{-3}, 10-270$, $15-166, \quad 48-61, \quad 34-389, \quad 254-432, \quad 1,000-2,000$ and $62-$ $250 \mathrm{pg} \mathrm{m}^{-3}$, respectively (Arinaitwe et al. 2018; Goel et al. 2016; Sakin and Tasdemir 2016; Nasir et al. 2014; Syed et al. 2013; Baek et al. 2010; Gioia et al. 2008; Zhang et al. 2008; Blanchard et al. 2006; Kim and Masunaga 2005). Hence, the values reported herein would be among the highest (comparable to those from India and Pakistan).

With regard to the total concentrations of PCBs in the dust, electronic waste, and sediments reported in other locations: Guangzhou and Hong Kong, Chennai, Northern Vietnam, Nakdong River (Korea), San Diego Bay, China, and Delhi (Anh et al. 2019, Chakraborty et al. 2016, Jin et al. 2012, Kumar et al. 2011, Li et al. 2012, Neira et al. 2018, Wang et al. 2013), it ranged from 4.02 to $114 \mathrm{ng} \mathrm{kg}^{-1}$, from 1.6 to 53 , from 0.25 to 14 , from 0.124 to 79.2 , from 23 to 1,387 , and from $<0.01$ to $99.40 \mathrm{ng} \mathrm{g}^{-1}$, respectively. Again, the values detected in this study would be among the highest reported in the literature.

Thus, the values found for Raipur would be higher than those found in Korea, China, and Delhi, and would only be exceeded by those detected in San Diego Bay, California.

Table 4. Correlation coefficient, $r(p=0.05)$, of PCBs in ambient air particulates

\begin{tabular}{|c|c|c|c|c|c|c|c|c|c|}
\hline Type & MCBs & DCBs & TCBs & TeCBs & $\mathrm{PeCBs}$ & $\mathrm{HCBs}$ & $\mathrm{HeCBs}$ & OCBs & $\mathrm{NCBs}$ \\
\hline MCBs & 1.00 & & & & & & & & \\
\hline DCBs & 0.51 & 1.00 & & & & & & & \\
\hline TCBs & 0.91 & 0.42 & 1.00 & & & & & & \\
\hline TeCBs & 0.79 & 0.91 & 0.62 & 1.00 & & & & & \\
\hline $\mathrm{PeCBs}$ & 0.65 & 0.89 & 0.72 & 0.84 & 1.00 & & & & \\
\hline HCBs & 0.27 & 0.83 & 0.42 & 0.60 & 0.90 & 1.00 & & & \\
\hline $\mathrm{HeCBs}$ & 0.93 & 0.76 & 0.90 & 0.89 & 0.89 & 0.61 & 1.00 & & \\
\hline OCBs & 0.86 & 0.64 & 0.96 & 0.74 & 0.89 & 0.66 & 0.96 & 1.00 & \\
\hline NCBs & 0.00 & 0.82 & -0.18 & 0.61 & 0.50 & 0.61 & 0.25 & 0.08 & 1.00 \\
\hline
\end{tabular}

Note: $\mathrm{MCBs}=$ monochlorobiphenyls; $\mathrm{DCBs}=$ dichlorobiphenyls; $\mathrm{TCBs}=$ trichlorobiphenyls; TeCBs $=$ tetrachlorobiphenyls; PCBs = pentrachlorobiphenyls; $\mathrm{HCBs}=$ hexachlorobiphenyls; $\mathrm{HeCBs}=$ heptachlorobiphenyls; $\mathrm{OCBs}=$ octachlorobiphenyls; and $\mathrm{NCBs}=$ nonachlorobiphenyls . 
Table 5. Comparison of PCB contamination

\begin{tabular}{|c|c|c|c|}
\hline Location & Sample type & Concentration & Reference \\
\hline London and Manchester, UK & Ambient air & $1,000-2,000 \mathrm{pg} \mathrm{m}^{-3}$ & Jaward et al. (2004) \\
\hline Coastal area, India & Ambient air & $216-1,077 \mathrm{pg} \mathrm{m}^{-3}$ & Zhang et al. (2008) \\
\hline Uludag University, Turkey & Ambient air & $293 \pm 257$ and $52 \pm 56 \mathrm{ng} \mathrm{m}^{-3}$ & Sakin and Tasdemir (2016) \\
\hline Lake Victoria, East Africa & Air & $65.6-244 \mathrm{pg} \mathrm{m}^{-3}$ & Arinaitwe et al. (2018) \\
\hline Yokohama, Japan & PM & $62-250 \mathrm{pg} \mathrm{m}^{-3}$ & Kim and Masunaga (2005) \\
\hline France & PM & $10-270 \mathrm{pg} \mathrm{m}^{-3}$ & Blanchard et al. (2006) \\
\hline Atlantic Ocean & PM & $4-220 \mathrm{pg} \mathrm{m}^{-3}$ & Gioia et al. (2008) \\
\hline Pohang, Korea & PM & $15-166 \mathrm{pg} \mathrm{m}^{-3}$ & Baek et al. (2010) \\
\hline Panjab, Pakistan & $\mathrm{PM}$ & $34-389 \mathrm{pg} \mathrm{m}^{-3}$ & Syed et al. (2013) \\
\hline Karachi, Lahore, Pakistan & PM & $48-61 \mathrm{pg} \mathrm{m}^{-3}$ & Nasir et al. (2014) \\
\hline Kanpur, India & PM & $254-432 \mathrm{pg} \mathrm{m}^{-3}$ & Goel et al. (2016) \\
\hline Guangzhou and Hong Kong & Outdoor dust & $\begin{array}{l}4.02-228 \mathrm{ng} \mathrm{kg}^{-1} \\
7.75-114 \mathrm{ng} \mathrm{kg}^{-1}\end{array}$ & Wang et al. (2013) \\
\hline Chennai, India & $\begin{array}{l}\text { Electronic waste: waste dismantling } \\
\text { sites, nearby highways, sub urban } \\
\text { and industrial site }\end{array}$ & $53,3.6,1.7$ and $1.6 \mathrm{ng} \mathrm{g}^{-1}$ & Chakraborty et al. (2016) \\
\hline Northern Vietnam & Street dust & 14,11 and $0.25 \mathrm{ng} \mathrm{g}^{-1}$ & Anh et al. (2019) \\
\hline Nakdong River, Korea & Sediment & $0.124-79.2 \mathrm{ng} \mathrm{g}^{-1}$ & Jin et al. (2012) \\
\hline San Diego Bay, Shelter Island Yacht & Sediment & $23-153,31-294$, and $151-1,387 \mathrm{ng} \mathrm{g}^{-1}$ & Neira et al. (2018) \\
\hline \multicolumn{4}{|l|}{$\begin{array}{l}\text { Basin (SIYB), Harbor Island West } \\
\text { (HW) and Harbor Island East (HE) }\end{array}$} \\
\hline China & Sediment & $126.49 \mathrm{ng} \mathrm{g}^{-1}$ & Li et al. (2012) \\
\hline Delhi & Soil & $<0.01-99.40 \mathrm{ng} \mathrm{g}^{-1}$ & Kumar et al. (2011) \\
\hline \multirow{3}{*}{ Raipur } & PM & $248 \pm 152 \mathrm{pg} \mathrm{m}^{-3}$ & Present work \\
\hline & Road dust & $241 \pm 146 \mathrm{ng} \mathrm{g}^{-1}$ & \\
\hline & Sediment & $328 \pm 99 \mathrm{ng} \mathrm{g}^{-1}$ & \\
\hline
\end{tabular}

These findings corroborate those reported by Chakraborty et al. (2013) in a study on atmospheric PCBs levels (gaseous and particulate phase) in the Indian cities: New Delhi, Agra, Kolkata, Mumbai, Goa, Chennai, and Bangalore. In view of the detected concentrations, the authors urge increasing control over the release of PCB sources in India and ask for measures to protect human health and the environment.

\section{Conclusions}

Very high concentrations of PCBs (mainly congeners 1 and 3) were detected in Raipur area: $143 \mathrm{pg} \mathrm{m}^{-3}\left(365 \mathrm{ng} \mathrm{g}^{-1}\right), 194$, and $299 \mathrm{ng} \mathrm{g}^{-1}$ for particulate matter, road dust, and sediments, respectively. These contents are among the highest ever reported in the literature, and clearly exceeded the recommended value of $60 \mathrm{ng} \mathrm{g}^{-1}$. The normalized total mean TEFs for the PM, road dust, and sediments from Raipur city were estimated at 0.00066 , 0.00058 , and 0.00047 , respectively. In view of the correlations with particulate matter, elemental carbon, organic carbon, and carbonate carbon, massive coal burning and vehicular emissions in Chhattisgarh region can be ascribed as the main sources of PCB pollution. Temporal evolution, tracked over an eight-year period, showed an average annual increment rate of approximately 5.4\% in PCBs concentration, while vertical profile analyses showed substantial PCBs concentrations at deeper sediments. Industrial uses, and coal and biomass combustion were apportioned as the major sources of PCBs contamination in the studied area. The collected data points to a dramatic situation, which calls for urgent action to meet the Stockholm Convention goals.

\section{Data Availability Statement}

All data, models, and code generated or used during the study appear in the published article.

\section{Acknowledgments}

The University Grant Commission, New Delhi, India is greatly acknowledged for the financial support through grant no. F.18-1/2011(BSR)2016.

\section{Supplemental Materials}

Table S1 is available online in the ASCE Library (www.ascelibrary .org).

\section{References}

Anh, H. Q., I. Watanabe, K. Tomioka, T. B. Minh, and S. Takahashi. 2019. "Characterization of 209 polychlorinated biphenyls in street dust from northern Vietnam: Contamination status, potential sources, and risk assessment." Sci. Total Environ. 652: 345-355. https://doi.org/10.1016/j scitotenv.2018.10.240.

Arinaitwe, K., D. C. G. Muir, B. T. Kiremire, P. Fellin, H. Li, C. Teixeira, and D. N. Mubiru. 2018. "Prevalence and sources of polychlorinated biphenyls in the atmospheric environment of Lake Victoria, East Africa." Chemosphere 193: 343-350. https://doi.org/10.1016/j.chemosphere .2017.11.041.

ATSDAR (Agency for Toxic Substances and Disease Registry). 2014. Case studies in environmental medicine, polychlorinated biphenyls (PCBs) toxicity. Atlanta: ATSDAR.

Baek, S. Y., S. D. Choi, H. Park, J. H. Kang, and Y. S. Chang. 2010. "Spatial and seasonal distribution of polychlorinated biphenyls (PCBs) in the vicinity of an iron and steel making plant." Environ. Sci. Technol. 44 (8): 3035-3040. https://doi.org/10.1021/es903251h.

Biterna, M., and D. Voutsa. 2005. "Polychlorinated biphenyls in ambient air of NW Greece and in particulate emissions." Environ. Int. 31 (5): 671-677. https://doi.org/10.1016/j.envint.2004.11.004.

Blanchard, M., M. J. Teil, and M. Chevreuil. 2006. "The seasonal fate of PCBs in ambient air and atmospheric deposition in northern France." 
J. Atmos. Chem. 53 (2): 123-144. https://doi.org/10.1007/s10874-006 -1321-z.

Brunciak, P. A., J. Dachs, T. P. Franz, C. L. Gigliotti, E. D. Nelson, B. J. Turpin, and S. J. Eisenreich. 2001. "Polychlorinated biphenyls and particulate organic/elemental carbon in the atmosphere of Chesapeake Bay, USA." Atmos. Environ. 35 (32): 5663-5677. https://doi.org/10.1016 /S1352-2310(01)00222-9.

Chakraborty, P., P. Balasubramanian, S. Sakthivel, and K. Bhupander. 2016. "Polychlorinated biphenyls in settled dust from informal electronic waste recycling workshops and nearby highways in urban centers and suburban industrial roadsides of Chennai city, India: Levels, congener profiles and exposure assessment." Sci. Total Environ. 573: 14131421. https://doi.org/10.1016/j.scitotenv.2016.07.129.

Chakraborty, P., G. Zhang, S. Eckhardt, J. Li, K. Breivik, P. K. S. Lam, S. Tanabe, and K. C. Jones. 2013. "Atmospheric polychlorinated biphenyls in Indian cities: Levels, emission sources and toxicity equivalents." Environ. Pollut. 182: 283-290. https://doi.org/10.1016/j.envpol.2013 .07 .032 .

Devi, N. L., I. C. Yadav, Q. Shihua, P. Chakraborty, and Y. Dan. 2014. "Distribution and risk assessment of polychlorinated biphenyls (PCBs) in the remote air and soil of Manipur, India." Environ. Earth Sci. 72 (10): 3955-3967. https://doi.org/10.1007/s12665-014-3284-8.

Du, S., T. J. Belton, and L. A. Rodenburg. 2008. "Source apportionment of polychlorinated biphenyls in the Tidal Delaware River." Environ. Sci. Technol. 42 (11): 4044-4051. https://doi.org/10.1021/es703047a.

Dumanoglu, Y., E. O. Gaga, E. Gungormus, S. C. Sofuoglu, and M. Odabasi. 2017. "Spatial and seasonal variations, sources, air-soil exchange, and carcinogenic risk assessment for PAHs and PCBs in air and soil of Kutahya, Turkey, the province of thermal power plants." Sci. Total Environ. 580: 920-935. https://doi.org/10.1016/j.scitotenv 2016.12.040.

Dyke, P. H., C. Foan, and H. Fiedler. 2003. "PCB and PAH releases from power stations and waste incineration processes in the UK." Chemosphere $50 \quad$ (4): 469-480. https://doi.org/10.1016/S0045 -6535(02)00627-6.

Froese, K. L., D. A. Verbrugge, S. A. Snyder, F. Tilton, M. Tuchman, A. Ostaszewski, and J. P. Giesy. 1997. "PCBs in the Detroit River water column.” J. Great Lakes Res. 23 (4): 440-449. https://doi.org/10.1016 /S0380-1330(97)70924-9.

Ganguly, R., D. Sharma, and P. Kumar. 2019. "Trend analysis of observational PM10 concentrations in Shimla city, India." Sustainable Cities Soc. 51: 101719. https://doi.org/10.1016/j.scs.2019 .101719.

Gioia, R., L. Nizzetto, R. Lohmann, J. Dachs, C. Temme, and K. C. Jones. 2008. "Polychlorinated biphenyls (PCBs) in air and seawater of the Atlantic Ocean: Sources, trends and processes." Environ. Sci. Technol. 42 (5): 1416-1422. https://doi.org/10.1021/es071432d.

Goel, A., K. Upadhyay, and M. Chakraborty. 2016. "Investigation of levels in ambient air near sources of polychlorinated biphenyls (PCBs) in Kanpur, India, and risk assessment due to inhalation." Environ. Monit. Assess. 188 (5): 278. https://doi.org/10.1007/s10661 -016-5280-9.

Guha, A., B. K. De, P. Dhar, T. Banik, M. Chakraborty, R. Roy, A. Choudhury, M. M. Gogoi, S. S. Babu, and K. K. Moorthy. 2015. "Seasonal characteristics of aerosol black carbon in relation to long range transport over Tripura in northeast India." Aerosol Air Qual. Res. 15 (3): 786-798. https://doi.org/10.4209/aaqr.2014.02.0029.

Han, Y. M., et al. 2015. "Elemental carbon and polycyclic aromatic compounds in a 150-year sediment core from Lake Qinghai, Tibetan Plateau, China: Influence of regional and local sources and transport pathways." Environ. Sci. Technol. 49 (7): 4176-4183. https://doi.org $/ 10.1021 / \mathrm{es} 504568 \mathrm{~m}$.

Han, Y. M., J. J. Cao, J. C. Chow, J. G. Watson, Z. S. An, and S. X. Liu. 2009. "Elemental carbon in urban soils and road dusts in Xi'an, China and its implication for air pollution." Atmos. Environ. 43 (15): 2464 2470. https://doi.org/10.1016/j.atmosenv.2009.01.040.

Harrad, S. 2009. Persistent organic pollutants, 288-292. Hoboken, NJ: Wiley-Blackwell.

Hesterberg, T. W., C. A. Lapin, and W. B. Bunn. 2008. "A comparison of emissions from vehicles fuelled with diesel or compressed natural gas."
Environ. Sci. Technol. 42 (17): 6437-6445. https://doi.org/10.1021 les071718i.

Hutzinger, O. 1974. The chemistry of PCB's. Boca Raton, FL: CRC Press. http://dx.doi.org/10.1201/9781351070591.

Ikonomou, M. G., M. P. Fernandez, W. Knapp, and P. Sather. 2002. "PCBs in Dungeness Crab reflect distinct source fingerprints among harbor/industrial sites in British Columbia." Environ. Sci. Technol. 36 (12): 2545-2551. https://doi.org/10.1021/es011209v.

Jaiswal, N. K., S. Ramteke, S. Chakradhari, K. S. Patel, H. Saathoff, S. Karnae, and K. John. 2019a. "Distribution, sources, and hazards of ambient carbonaceous particulates in central india." J. Hazard. Toxic Radioact. Waste 23 (4): 05019002. https://doi.org/10.1061/(ASCE)HZ .2153-5515.0000447.

Jaiswal, N. K., S. Ramteke, K. S. Patel, H. Saathoff, S. Nava, F. Lucarelli, E. Yubero, and M. Viana. 2019b. "Winter particulate pollution over Raipur, India." J. Hazard. Toxic Radioact. Waste 23 (4): 05019001. https://doi.org/10.1061/(ASCE)HZ.2153-5515.0000444.

Jaward, F. M., N. J. Farrar, T. Harner, A. J. Sweetman, and K. C. Jones. 2004. "Passive air sampling of PCBs, PBDEs, and organochlorine pesticides across Europe.” Environ. Sci. Technol. 38 (1): 34-41. https://dx .doi.org/10.1021/es034705n.

Jin, R., S. U. Park, J. E. Park, and J.-G. Kim. 2012. "Polychlorinated biphenyl congeners in river sediments: Distribution and source identification using multivariate factor analysis." Arch. Environ. Contam. Toxicol. 62 (3): 411-423. https://doi.org/10.1007/s00244-011-9722-7.

Kim, K. S., and S. Masunaga. 2005. "Behavior and source characteristic of PCBS in urban ambient air of Yokohama, Japan." Environ. Pollut. 138 (2): 290-298. https://doi.org/10.1016/j.envpol.2005.03.011.

Kodavanti, P. R. S., J. Valdez, J.-H. Yang, M. Curras-Collazo, and B. G. Loganathan. 2017. "Polychlorinated biphenyls, polybrominated biphenyls, polychlorinated dibenzo-p-dioxins, and polychlorinated dibenzofurans reproductive and developmental toxicology." Chap. 39 in Reproductive and developmental toxicology. 2nd ed. edited by R. C. Gupta, 711-743. Cambridge, MA: Academic Press.

Kumar, B., S. Kumar, G. Goel, R. Gaur, M. Mishra, S. K. Singh, D. Prakash, P. Chakraborty, and C. S. Sharma. 2011. "Distribution of polychlorinated biphenyls in agricultural soils from NCR, Delhi, India." Ann. Biol. Res. 2 (3): 247-254.

Laroo, C. A., C. R. Schenk, L. J. Sanchez, J. McDonald, and P. L. Smith. 2012. "Emissions of PCDD/Fs, PCBs, and PAHs from legacy on-road heavy-duty diesel engines." Chemosphere 89 (11): 1287-1294. https:// doi.org/10.1016/j.chemosphere.2012.05.022.

Lee, R. G. M., P. Coleman, J. L. Jones, K. C. Jones, and R. Lohmann. 2005. "Emission factors and importance of PCDD/Fs, PCBs, PCNs, PAHs and $\mathrm{PM}_{10}$ from the domestic burning of coal and wood in the UK." Environ. Sci. Technol. 39 (6): 1436-1447. https://doi.org/10.1021 les048745i.

Li, W.-H., Y.-Z. Tian, G.-L. Shi, C.-S. Guo, Y.-C. Feng, and X.-P. Yue. 2012. "Source and risk assessment of PCBs in sediments of Fenhe reservoir and watershed, China." J. Environ. Monit. 14 (4): 1256-1263. https://doi.org/10.1039/c2em10983b.

Liu, R., S. Ma, G. Li, Y. Yu, and T. An. 2019. "Comparing pollution patterns and human exposure to atmospheric PBDEs and PCBs emitted from different e-waste dismantling processes." J. Hazard. Mater. 369: 142-149. https://doi.org/10.1016/j.jhazmat.2019.02.029.

Malina, N., and E. A. Mazlova. 2017. "Temporal and spatial variation of polychlorinated biphenyls (PCBs) contamination in environmental compartments of highly polluted area in Central Russia." Chemosphere 185: 227-236. https://doi.org/10.1016/j.chemosphere .2017.06.137.

Mao, S., G. Zhang, S. Zhao, J. Li, X. Liu, Z. Cheng, G. Zhong, R. N. Mali, and X. Liu. 2019. "High abundance of unintentionally produced tetrachlorobiphenyls (PCB47/48/75, 51, and 68) in the atmosphere at a regional background site in east China." Environ. Sci. Technol. 53 (7): 3464-3470. https://doi.org/10.1021/acs.est.8b07286.

Meijer, S. N., W. A. Ockenden, A. Sweetman, K. Breivik, J. O. Grimalt, and K. C. Jones. 2003. "Global distribution and budget of PCBs and HCB in background surface soils: implications for sources and environmental processes." Environ. Sci. Technol. 37 (4): 667-672. https://doi .org/10.1021/es0258091. 
Na, K., Y. P. Kim, I. Moon, and K.-C. Moon. 2004. "Chemical composition of major VOC emission sources in the Seoul atmosphere." Chemosphere 55 (4): 585-594. https://doi.org/10.1016/j.chemosphere .2004.01.010.

Nasir, J., X. Wang, B. Xu, C. Wang, D. R. Joswiak, S. Rehman, A. Lodhi, S. Shafiq, and R. Jilani. 2014. "Selected organochlorine pesticides and polychlorinated biphenyls in urban atmosphere of Pakistan: Concentration, spatial variation and sources." Environ. Sci. Technol. 48 (5): 2610-2618. https://doi.org/10.1021/es404711n.

Neira, C., M. Vales, G. Mendoza, E. Hoh, and L. A. Levin. 2018. "Polychlorinated biphenyls (PCBs) in recreational marina sediments of San Diego Bay, southern California." Mar. Pollut. Bull. 126: 204 214. https://doi.org/10.1016/j.marpolbul.2017.10.096.

Ozdemir, H., L. Pozzoli, T. Kindap, G. Demir, B. Mertoglu, N. Mihalopoulos, C. Theodosi, M. Kanakidou, U. Im, and A. Unal. 2014. "Spatial and temporal analysis of black carbon aerosols in Istanbul megacity." Sci. Total Environ. 473-474: 451-458. https://doi .org/10.1016/j.scitotenv.2013.11.102.

Patel, K. S., Y. Nayak, and C.-C. Hung. 2013. "Polychlorinated biphenyls contamination in pond sediment of central India." In Water \& Environmental Dynamics Proc., 6th Int. Conf. on Water Resources and Environment Research, 588-605. Berlin, Germany: epubli GmbH.

Patel, K. S., S. Ramteke, B. L. Sahu, Y. Nayak, S. Sharma, and C. Hung. 2015. "Polychlorinated biphenyls contamination of sludge in India." Am. J. Anal. Chem. 6 (11): 867-877. https://doi.org/10.4236/ajac .2015.611082.

Robertson, L. W., and L. G. Hansen. 2001. "PCBs: Recent advances in environmental toxicology and health effects." Med. Health Sci. 8: 496.

Sakin, A. E., and Y. Tasdemir. 2016. "Determination of atmospheric PCB level variations in continuously collected samples." Arch. Environ. Contam. Toxicol. 71 (2): 235-245. https://doi.org/10.1007/s00244 -016-0291-7.

Sericano, J. L. 2002. "Quantitative determination of polychlorinated biphenyls by gas chromatography/mass spectrometry using the selected ion monitoring mode." Geochemical and Environmental Research Group Standard Operating Procedures, SOP-0205.

Shyu, G.-S., B.-Y. Cheng, C.-T. Chiang, P.-H. Yao, and T.-K. Chang. 2011. "Applying factor analysis combined with kriging and information entropy theory for mapping and evaluating the stability of groundwater quality variation in Taiwan.” Int. J. Environ. Res. Public Health 8 (4): 1084-1109. https://doi.org/10.3390/ijerph8041084.

Simpson, S., and G. Batley. 2016. Sediment quality assessment: A practical guide. 2nd ed. Clayton South, VIC, Australia: CSIRO Publishing.

Syed, J. H., R. N. Malik, J. Li, G. Zhang, and K. C. Jones. 2013. "Levels, distribution and air-soil exchange fluxes of polychlorinated biphenyls (PCBs) in the environment of Punjab Province, Pakistan.” Ecotoxicol. Environ. Saf. 97: 189-195. https://doi.org/10.1016/j.ecoenv.2013.06.005.

Tanabe, S., S. N. Kannan, T. Wakimato, and R. Tatsukawa. 1987. "Method for determination of three toxic non-ortho chlorine substituted coplanar PCBs in environmental samples at part-per-trillion levels." Int. J. Environ. Anal. Chem. 29 (3): 199-213. https://doi.org/10.1080 /03067318708079836.

United Nations Environmental Programme, UNITAR. 2017. "PCB: A forgotten legacy? 2028: Final elimination of PCB". http://www.pops.int /Portals/0/download.aspx?d=UNEP-POPS-PCB-Brochure-PEN-2017 .English.pdf.

USEPA. 2010. Recommended toxicity equivalence factors (TEFs) for human health risk assessments of 2,3,7,8-tetrachlorodibenzo-p-dioxin and dioxinlike compounds. EPA/100/R 10/005. Washington, DC: USEPA.

Wade, T. L., E. L. Atlas, J. M. Brooks, M. C. Kennicutt, R. G. Fox, J. Sericano, B. Garcia-Romero, and D. Defreitas. 1988. "NOAA Gulf of Mexico status and trends program: Trace organic contaminant distribution in sediments and oysters." Estuaries 11 (3): 171-179. https://doi .org/10.2307/1351969.

Wang, W., M. J. Huang, J. S. Zheng, K. C. Cheung, and M. H. Wong. 2013. "Exposure assessment and distribution of polychlorinated biphenyls (PCBs) contained in indoor and outdoor dusts and the impacts of particle size and bio-accessibility." Sci. Total Environ. 463-464: 1201-1209. https://doi.org/10.1016/j.scitotenv.2013.04.059.

Weber, R., F. Lino, T. Imagawa, M. Takeuchi, T. Sakurai, and M. Sadakata. 2001. "Formation of PCDF, PCDD, PCB and PCN in de novo synthesis from PAH: Mechanistic aspects and correlation to fluidized bed incinerators." Chemosphere 44 (6): 1429-1438. https://doi.org /10.1016/S0045-6535(00)00508-7.

Yang, Y., and W. Baumann. 1996. "Study of polychlorinated biphenyls in street dust by supercritical fluid extraction-gas chromatography/mass spectrometry." Fresenius J. Anal. Chem. 354 (1): 56-60. https://doi .org/10.1007/s002169600009.

Zhang, G., P. Chakraborty, J. Li, P. Sampathkumar, T. Balasubramanian, K. Kandasamy, S. Takahashi, S. Annamalai, S. Tanabe, and K. C. Jones. 2008. "Passive atmospheric sampling of organochlorine pesticides, polychlorinated biphenyls, and polybrominated diphenyl ethers in urban, rural, and wetland sites along the coastal length of India." Environ. Sci. Technol. 42 (22): 8218-8823. https://doi.org/10.1021 Yes8016667.

Zong, Y., Q. Xiao, and S. Lu. 2016. "Black carbon (BC) of urban topsoil of steel industrial city (Anshan), Northeastern China: Concentration, source identification and environmental implication." Sci. Total Environ. 569570: 990-996. https://doi.org/10.1016/j.scitotenv.2016.06.097. 